\title{
Two-loop five-point amplitudes of super Yang-Mills and supergravity in pure spinor superspace
}

\author{
Carlos R. Mafra ${ }^{a}$ and Oliver Schlotterer ${ }^{b}$ \\ ${ }^{a}$ DAMTP, University of Cambridge, \\ Wilberforce Road, Cambridge, CB3 0WA, U.K. \\ ${ }^{b}$ Max-Planck-Institut für Gravitationsphysik Albert-Einstein-Institut, \\ 14476 Potsdam, Germany \\ E-mail: c.r.mafra@damtp.cam.ac.uk, olivers@aei.mpg.de
}

\begin{abstract}
Supersymmetric integrands for the two-loop five-point amplitudes in tendimensional super Yang-Mills and type II supergravity are proposed. The kinematic numerators are manifestly local and satisfy the duality between color and kinematics described by Bern, Carrasco and Johansson. Our results are expected to reproduce the integrated two-loop amplitudes in dimensions $D<7$. The UV divergence in the critical dimension $D=7$ matches the low-energy limit of the corresponding superstring amplitudes and is written in terms of SYM tree amplitudes.
\end{abstract}

KEYwords: Superstrings and Heterotic Strings, Superspaces, Field Theories in Higher Dimensions, BRST Symmetry

ARXIV EPRINT: 1505.02746 


\section{Contents}

1 Introduction $\quad 2$

2 Review 3

2.1 BCJ duality between color and kinematics 3

2.2 Ten-dimensional SYM 4

2.3 Multiparticle superfields 4

2.4 Pure spinor superspace 5

3 Field-theory amplitudes and BRST cohomology at two-loops $\quad 6$

$\begin{array}{lll}3.1 & \text { BRST properties of kinematic numerators } & 7\end{array}$

$\begin{array}{lll}3.2 & \text { The SYM two-loop four-point amplitude } & 7\end{array}$

$\begin{array}{lll}3.3 & \text { Five-point building blocks in pure spinor superspace } & 8\end{array}$

4 The two-loop five-point amplitudes in SYM and supergravity 9

$\begin{array}{llr}4.1 \text { Color factors } & 9\end{array}$

$\begin{array}{lll}4.2 & \text { Planar cubic graphs and superspace numerators } & 10\end{array}$

$\begin{array}{lll}4.3 & \text { Planar two-loop amplitudes in SYM } & 11\end{array}$

$\begin{array}{lll}4.4 & \text { Non-planar cubic graphs and superspace numerators } & 12\end{array}$

$\begin{array}{ll}\text { 4.5 Non-planar two-loop amplitudes in SYM } & 14\end{array}$

$\begin{array}{lll}4.6 & \text { Two-loop amplitudes in supergravity } & 14\end{array}$

5 UV divergences $\quad \mathbf{1 5}$

5.1 Two-loop UV divergences in SYM 15

$\begin{array}{lll}5.2 & \text { Two-loop UV divergences in supergravity } & 16\end{array}$

$\begin{array}{ll}5.3 \text { UV divergence and R-symmetry } & 17\end{array}$

6 Conclusion and outlook $\quad 18$

$\begin{array}{ll}\text { A Diagrammatic bookkeeping of BRST variations } & 19\end{array}$

$\begin{array}{ll}\text { B Comparison with the four-dimensional solution } & 21\end{array}$

C One-loop UV divergences $\quad 23$

C.1 One-loop UV divergences in SYM 23

C.2 One-loop UV divergences in supergravity 24 


\section{Introduction}

Tree-level and one-loop scattering amplitudes of ten-dimensional super Yang-Mills (SYM) have been recently determined using a method based on two fundamental principles: locality and BRST invariance [1-3]. Locality refers to the expansion of amplitudes in terms of cubic graphs with definite propagator structure [4, 5], and BRST invariance is a property of pure spinor superspace that guarantees manifest supersymmetry and gauge invariance [6]. Even though the notion of BRST invariance is motivated by the pure spinor formalism of the superstring [6], pure spinor variables are long known to simplify the description of ten-dimensional SYM [7, 8], as will be corroborated once more by this paper.

In the subsequent, we will describe the two-loop extension of this method and use it to derive the five-point two-loop integrand of ten-dimensional SYM in an intuitive manner. In doing so, we follow closely the organization found in a beautiful paper by Carrasco and Johansson [9] which makes the symmetry between color and kinematic degrees of freedom $[4,5]$ manifest and thereby leads to the supergravity integrand without any extra effort [10]. As an additional benefit of the pure spinor superspace representation, the kinematic numerators are manifestly local due to the very nature of our method (bypassing the inverse Gram determinants in [9]) and do not require any constructive input from unitarity.

As the main result of this paper, the color-dressed five-point two-loop amplitude of SYM in ten dimensions will be explicitly constructed in (4.15), following the guidelines of the method described in section 3. The polarization dependence is furnished by two local kinematic building blocks $T_{12,3 \mid 4,5}$ and $T_{1,2,3 \mid 4,5}^{m}$ written in pure spinor superspace and inspired by string theory whose bosonic components can be downloaded from [11]. Thanks to their compatibility with the Bern-Carrasco-Johansson (BCJ) duality between color and kinematics $[4,5]$, the corresponding supergravity amplitude is a straightforward corollary [10] and given in (4.16).

Since our results are formulated in ten dimensions, standard dimensional reduction gives rise to their lower-dimensional counterparts [12]. In section 5, the UV divergences of maximally supersymmetric SYM and supergravity in the two-loop critical dimension $D=7$ are written in terms of SYM tree amplitudes. As a consistency check [13], the superspace expression for the supergravity UV divergence in (5.9) matches the low-energy limit of the closed-string amplitude [14]. However, type IIB superstring two-loop amplitudes violate the U(1) R-symmetry as a consequence of S-duality [14-17] while supergravity and its two-loop UV divergence in $D=7$ dimensions conserve it. This apparent paradox is resolved by the prefactor $(7-D)$ in the R-symmetry violating components that appears once the ten-dimensional superstring kinematic factor is dimensionally reduced to involve a $D$-dimensional dilaton state. The same mechanism applies to the one-loop UV divergence, where a prefactor of $(8-D)$ along with a $D$-dimensional dilaton reconciles Sduality properties of the superstring amplitude $[14,18]$ with R-symmetry of supergravity, see appendix C. 


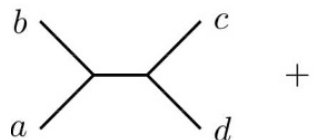

$C_{i}$

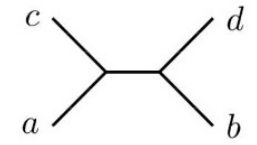

$C_{j}$

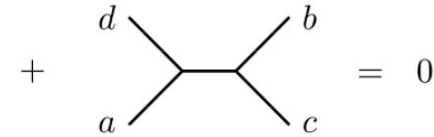

$C_{k}$

Figure 1. The Jacobi identity implies the vanishing of the color factors associated to a triplet of cubic graphs, $C_{i}+C_{j}+C_{k}=0$. In the above diagrams, the legs $a, b, c$ and $d$ may represent arbitrary subdiagrams. The BCJ duality states that their corresponding kinematic numerators $N_{i}(\ell)$ can be chosen such that $N_{i}(\ell)+N_{j}(\ell)+N_{k}(\ell)=0$.

\section{Review}

\subsection{BCJ duality between color and kinematics}

Bern, Carrasco and Johansson (BCJ) proposed an organization scheme for gauge theory amplitudes based on cubic vertices where color and kinematic degrees of freedom enter on completely symmetric footing $[4,5]$ :

$$
\mathcal{A}_{n}^{g-\text { loop }}=\int \prod_{j=1}^{g} \frac{d^{D} \ell_{j}}{(2 \pi)^{D}} \sum_{\Gamma_{i}} \frac{N_{i}(\ell) C_{i}}{\prod_{k} P_{k, i}(\ell)}
$$

The sum is understood to encompass all "cubic" graphs $\Gamma_{i}$ with $n$ external legs, $g$ loops and only trivalent vertices as well as appropriate symmetry factors to avoid overcounting. The propagators $P_{k, i}(\ell)$ refer to the squared momentum in the $k^{\text {th }}$ internal line of the $i^{\text {th }}$ graph. The color tensors $C_{i}$ are obtained by dressing each vertex of $\Gamma_{i}$ with a factor of $f^{a b c}$, the structure constants of the gauge group, and each internal line by $\delta^{a b}$. Finally, the kinematic numerators $N_{i}(\ell)$ encode the dependence on polarizations and (external or internal) momenta $k, \ell$. They furnish the only ingredient of (2.1) that cannot be immediately read off from the graphs, and they will be in the main focus of this work.

Triplets of color tensors $C_{i}, C_{j}, C_{k}$ associated with diagrams $i, j, k$ that differ in only one propagator (see figure 1) vanish due to the Jacobi identity

$$
f^{a b e} f^{c d e}+f^{b c e} f^{a d e}+f^{c a e} f^{b d e}=0
$$

valid for any gauge group. The BCJ conjecture $[4,5]$ states that amplitudes in (2.1) can be represented such that for any vanishing color triplet $C_{i}+C_{j}+C_{k}$, the corresponding kinematic decorations $N_{i}(\ell)+N_{j}(\ell)+N_{k}(\ell)$ of diagrams $i, j, k$ vanish as well. This statement is illustrated in figure 1 and understood to hold for any value of the loop momenta $\ell$. Of course, the momentum in the four external lines represented by $a, b, c, d$ and the subdiagrams beyond them have to be the same in the three graphs $i, j, k$.

Once a gauge theory amplitude (2.1) has been cast into such a "BCJ form",

$$
C_{i}+C_{j}+C_{k}=0 \Rightarrow N_{i}(\ell)+N_{j}(\ell)+N_{k}(\ell)=0
$$


then the corresponding gravity amplitude follows from trading color tensors for a second copy of the kinematic numerators, $C_{i} \rightarrow \tilde{N}_{i}(\ell)[10]$

$$
\mathcal{M}_{n}^{g-\text { loop }}=\int \prod_{j=1}^{g} \frac{d^{D} \ell_{j}}{(2 \pi)^{D}} \sum_{\Gamma_{i}} \frac{N_{i}(\ell) \tilde{N}_{i}(\ell)}{\prod_{k} P_{k, i}(\ell)} .
$$

The second copy of $\tilde{N}_{i}(\ell)$ does not need to stem from the same gauge theory or satisfy the kinematic Jacobi identities (2.3). The polarization tensors of the resulting gravity amplitudes (2.4) are then given by the tensor products of the gauge theory polarizations contained in $N_{i}(\ell) \tilde{N}_{i}(\ell)$. We will apply this double-copy construction to the two-loop fivepoint amplitudes in ten-dimensional SYM and the resulting type II supergravities, see section 4.6. When dressed with non-supersymmetric numerators $\tilde{N}_{i}(\ell)$ of pure Yang-Mills, our results for $N_{i}(\ell)$ might serve as a convenient starting point to investigate two-loop five-point amplitudes in half-maximal supergravity.

\subsection{Ten-dimensional SYM}

Ten-dimensional SYM can be described in a super-Poincaré covariant manner using a set of superfields $\left\{A_{\alpha}, A^{m}, W^{\alpha}, F_{m n}\right\}$ [19]. They depend on the ten-dimensional superspace coordinates $\left\{x^{m}, \theta^{\alpha}\right\}$ with vector and spinor indices $m, n=0,1, \ldots, 9$ and $\alpha, \beta=1,2, \ldots, 16$ of the Lorentz group. The linearized equations of motion [19] in momentum space ${ }^{1}$

$$
\begin{aligned}
D_{\alpha} A_{\beta}+D_{\beta} A_{\alpha} & =\gamma_{\alpha \beta}^{m} A_{m}, & D_{\alpha} A_{m} & =\left(\gamma_{m} W\right)_{\alpha}+k_{m} A_{\alpha} \\
D_{\alpha} F_{m n} & =2 k_{[m}\left(\gamma_{n]} W\right)_{\alpha}, & D_{\alpha} W^{\beta} & =\frac{1}{4}\left(\gamma^{m n}\right)_{\alpha}{ }^{\beta} F_{m n}
\end{aligned}
$$

involve light-like momenta $k^{2}=0$ via plane waves $e^{k \cdot x}$ and fermionic covariant derivatives

$$
D_{\alpha} \equiv \frac{\partial}{\partial \theta^{\alpha}}+\frac{1}{2}\left(\gamma^{m} \theta\right)_{\alpha}, \quad\left\{D_{\alpha}, D_{\beta}\right\}=\gamma_{\alpha \beta}^{m} \partial_{m}
$$

with $16 \times 16$ Pauli matrices ${ }^{2} \gamma_{\alpha \beta}^{m}=\gamma_{\beta \alpha}^{m}$ subject to the Clifford algebra $\gamma_{\alpha \beta}^{(m} \gamma^{n) \beta \gamma}=\eta^{m n} \delta_{\alpha}^{\gamma}$.

The equations of motion (2.5) identify $8+8$ on-shell degrees of freedom associated with a gluon and gluino. Their polarizations are described by a transverse vector $k_{m} e^{m}=0$ and a spinorial solution to the massless Dirac-equation $k_{m} \gamma_{\alpha \beta}^{m} \chi^{\beta}=0$, respectively. In a gauge where $\theta^{\alpha} A_{\alpha}=0$, they enter the explicit $\theta$-expansions via [20-22]

$$
A_{\alpha}=e^{k \cdot x}\left(\frac{1}{2} e_{m}\left(\gamma^{m} \theta\right)_{\alpha}-\frac{1}{3}\left(\chi \gamma_{m} \theta\right)\left(\gamma^{m} \theta\right)_{\alpha}-\frac{1}{16} k_{m} e_{n}\left(\gamma_{p} \theta\right)_{\alpha}\left(\theta \gamma^{m n p} \theta\right)+\cdots\right) .
$$

\subsection{Multiparticle superfields}

Ten-dimensional SYM arises from the massless sector of the open pure spinor superstring [6]. Its $i^{\text {th }}$ scattering state is represented by the integrated vertex operator $U_{i}$ which involves all of $\left\{A_{\alpha}^{i}, A_{i}^{m}, W_{i}^{\alpha}, F_{m n}^{i}\right\}$ along with various worldsheet fields. In string

\footnotetext{
${ }^{1}$ Our conventions for (anti-)symmetrizing $n$ vector indices include a factor of $1 / n$ !.

${ }^{2}$ Pauli matrices frequently appear in their antisymmetrized combinations with symmetry properties $\gamma_{\alpha \beta}^{m n p}=-\gamma_{\beta \alpha}^{m n p}$ and $\gamma_{\alpha \beta}^{m n p q r}=\gamma_{\beta \alpha}^{m n p q r}$ which are normalized like $\gamma_{\alpha}^{m n} \equiv \frac{1}{2}\left(\gamma^{m} \gamma^{n}-\gamma^{n} \gamma^{m}\right)_{\alpha}^{\beta}$.
} 

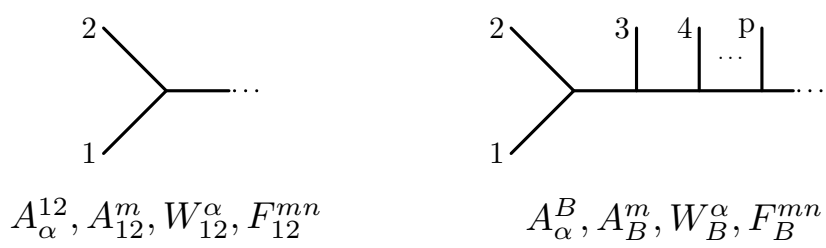

Figure 2. Interpretation of multiparticle superfields as tree-level subdiagrams where the external states are represented by multiparticle labels $B=12 \ldots p$.

calculations, the latter act on additional vertex operators via operator product expansions (OPEs) and build up so-called multiparticle superfields [23]

$$
\begin{aligned}
A_{12}^{\alpha} & \equiv-\frac{1}{2}\left[A_{\alpha}^{1}\left(k^{1} \cdot A^{2}\right)+A_{m}^{1}\left(\gamma^{m} W^{2}\right)_{\alpha}-(1 \leftrightarrow 2)\right] \\
A_{m}^{12} & \equiv \frac{1}{2}\left[A_{p}^{1} F_{p m}^{2}-A_{m}^{1}\left(k^{1} \cdot A^{2}\right)+\left(W^{1} \gamma_{m} W^{2}\right)-(1 \leftrightarrow 2)\right] \\
W_{12}^{\alpha} & \equiv \frac{1}{4}\left(\gamma^{m n} W^{2}\right)^{\alpha} F_{m n}^{1}+W_{2}^{\alpha}\left(k^{2} \cdot A^{1}\right)-(1 \leftrightarrow 2) \\
F_{12}^{m n} & \equiv F_{2}^{m n}\left(k^{2} \cdot A^{1}\right)+F_{2}^{[m}{ }_{p} F_{1}^{n] p}+k_{12}^{[m}\left(W_{1} \gamma^{n]} W_{2}\right)-(1 \leftrightarrow 2) .
\end{aligned}
$$

In the point-particle limit, these string-inspired generalizations of linearized SYM accompany the tree-level subdiagrams seen in figure 2. They capture, for instance, the reducible parts of the subsequent five-point two-loop amplitudes and have generalizations $\left\{A_{\alpha}^{12 \ldots p}\right.$, $\left.A_{12 \ldots p}^{m}, W_{12 \ldots p}^{\alpha}, F_{m n}^{12 \ldots p}\right\}$ to any number of states [23], see figure 2. We will use multiparticle labels such as $B=12 \ldots p$ to keep the multiplicity of superfields unspecified, i.e. $W_{B}^{\alpha}$ can become either $W_{1}^{\alpha}$ or $W_{12}^{\alpha}$ upon setting $B=1$ or $B=12$.

The non-linear version of the equations of motion (2.5) are solved by the generating series of multiparticle fields [24]. The non-linearities are reflected by the contact terms $\sim\left(k^{1} \cdot k^{2}\right)$ in their multiparticle equations of motion

$$
\begin{aligned}
2 D_{(\alpha} A_{\beta}^{12} & =\gamma_{\alpha \beta}^{m} A_{m}^{12}+\left(k^{1} \cdot k^{2}\right)\left(A_{\alpha}^{1} A_{\beta}^{2}+A_{\beta}^{1} A_{\alpha}^{2}\right) \\
D_{\alpha} A_{m}^{12} & =\left(\gamma_{m} W^{12}\right)_{\alpha}+k_{m}^{12} A_{\alpha}^{12}+\left(k^{1} \cdot k^{2}\right)\left(A_{\alpha}^{1} A_{m}^{2}-A_{\alpha}^{2} A_{m}^{1}\right) \\
D_{\alpha} W_{12}^{\beta}= & \frac{1}{4}\left(\gamma^{m n}\right)_{\alpha}^{\beta} F_{m n}^{12}+\left(k^{1} \cdot k^{2}\right)\left(A_{\alpha}^{1} W_{2}^{\beta}-A_{\alpha}^{2} W_{1}^{\beta}\right) \\
D_{\alpha} F_{m n}^{12}= & k_{m}^{12}\left(\gamma_{n} W^{12}\right)_{\alpha}-k_{n}^{12}\left(\gamma_{m} W^{12}\right)_{\alpha}+\left(k^{1} \cdot k^{2}\right)\left(A_{\alpha}^{1} F_{m n}^{2}-A_{\alpha}^{2} F_{m n}^{1}\right) \\
& +\left(k^{1} \cdot k^{2}\right)\left(A_{n}^{1}\left(\gamma_{m} W^{2}\right)_{\alpha}-A_{n}^{2}\left(\gamma_{m} W^{1}\right)_{\alpha}-A_{m}^{1}\left(\gamma_{n} W^{2}\right)_{\alpha}+A_{m}^{2}\left(\gamma_{n} W^{1}\right)_{\alpha}\right),
\end{aligned}
$$

where the notation $k_{12 \ldots p}^{m} \equiv k_{1}^{m}+k_{2}^{m}+\ldots+k_{p}^{m}$ will be used throughout this work.

\subsection{Pure spinor superspace}

The physical components of superspace expressions in SYM can be conveniently extracted using a bosonic pure spinor $\lambda^{\alpha}$ which is defined to obey $[7,8]$

$$
\left(\lambda \gamma^{m} \lambda\right)=0
$$

Corollaries of this pure spinor constraint include $\left(\lambda \gamma^{m}\right)_{\alpha}\left(\lambda \gamma_{m}\right)_{\beta}=0=\left(\lambda \gamma^{m} \gamma^{p q} \lambda\right)$ which will frequently enter the subsequent manipulations. Pure spinor superspace is defined by an 
extension of the standard ten-dimensional superspace coordinates $\left\{x^{m}, \theta^{\alpha}\right\}$ to $\left\{x^{m}, \theta^{\alpha}, \lambda^{\alpha}\right\}$ subject to the following component prescription [6]

$$
\left\langle\left(\lambda \gamma^{m} \theta\right)\left(\lambda \gamma^{n} \theta\right)\left(\lambda \gamma^{p} \theta\right)\left(\lambda \gamma_{m n p} \theta\right)\right\rangle=2880 .
$$

The order $\lambda^{3} \theta^{5}$ is singled out by supersymmetry and the cohomology of the BRST operator

$$
Q \equiv \lambda^{\alpha} D_{\alpha}
$$

BRST-closed superfields $Q S(\theta, \lambda)=0$ give rise to supersymmetric and gauge invariant components $\langle S(\theta, \lambda)\rangle$ under (2.11) whereas BRST-exact superfields $E(\theta, \lambda)=Q \Sigma(\theta, \lambda)$ yield $\langle E(\theta, \lambda)\rangle=0$ [6]. Expressions of order $\lambda^{3} \theta^{5}$ with a different tensor structure as compared to (2.11) are uniquely fixed by group theory since this tensor product contains only one Lorentz scalar. The required group theory manipulations are automated in [25].

As the central idea of this work and preceding papers [1-3], scattering amplitudes $\langle S(\theta, \lambda)\rangle$ in ten-dimensional SYM are proposed by constructing a BRST-invariant superfield $S(\theta, \lambda)$ whose kinematic poles reproduce the expected Feynman diagrams. In this approach, the superfield $S(\theta, \lambda)$ carries the kinematical data of state $i$ through the superfields $\left\{A_{\alpha}^{i}, A_{i}^{m}, W_{i}^{\alpha}, F_{m n}^{i}\right\}$ whose equations of motion (2.5) determine the BRST variation.

For example, the unintegrated vertex operator of the superstring,

$$
V_{i} \equiv \lambda^{\alpha} A_{\alpha}^{i}, \quad Q V_{i}=0
$$

suffices to write down the three-point tree-level subamplitude in pure spinor superspace,

$$
A^{\text {tree }}(1,2,3)=\left\langle V_{1} V_{2} V_{3}\right\rangle=\left(e_{1} \cdot e_{2}\right)\left(e_{3} \cdot k_{1}\right)+e_{1}^{m}\left(\chi_{2} \gamma_{m} \chi_{3}\right)+\operatorname{cyc}(1,2,3) .
$$

This sample calculation based on the $\theta$-expansion (2.7) and the prescription (2.11) illustrates how all the component amplitudes are supersymmetrically embedded into BRSTclosed superfields such as $V_{1} V_{2} V_{3}$. We will limit our subsequent discussion of two-loop amplitudes to their superspace representatives since the component extraction for any superfield numerator can be performed in an automated way [25, 26], and the resulting components can be downloaded from [11].

\section{Field-theory amplitudes and BRST cohomology at two-loops}

Multiloop superstring amplitudes computed with the pure spinor formalism give rise to superspace expressions in the cohomology of the pure spinor BRST charge [27, 28]. As explained in [6], superfields in the BRST cohomology translate to gauge invariant and supersymmetric component expansions. Since ten-dimensional SYM and type II supergravities arise in a certain limit of superstring theories, their scattering amplitudes belong to the BRST cohomology as well. Multiloop integrands for these field-theory amplitudes are strongly constrained by demanding BRST invariance and the propagator structure expected from Feynman diagrams. Together with a string-inspired set of admissible kinematic building blocks, these requirements will allow us to fix the two-loop five-point amplitudes. 


\subsection{BRST properties of kinematic numerators}

Inspired by the discussion of section 2.1, multiloop amplitudes of ten-dimensional SYM theory are organized in terms of cubic graphs

$$
\mathcal{A}_{n}^{g-\text { loop }}=\int \prod_{j=1}^{g} \frac{d^{D} \ell_{j}}{(2 \pi)^{D}} \sum_{\Gamma_{i}} \frac{\left\langle N_{i}(\ell)\right\rangle C_{i}}{\prod_{k} P_{k, i}(\ell)} .
$$

Maximal supersymmetry suppresses any graph $\Gamma_{i}$ with a triangle, bubble or tadpole subdiagram [29]. In the superspace setup of this paper, the numerators $N_{i}(\ell)$ will be given by local pure spinor superspace expressions, whose form is suggested by the propagator structure $P_{k, i}(\ell)$ of its corresponding cubic graph. Requiring BRST invariance of the integrand in (3.1) largely determines the mapping between cubic graphs and superspace numerators, and the subsequent examples are completely fixed when assuming a string-inspired ansatz for admissible kinematic building blocks.

If individual numerators are not BRST invariant by themselves, their BRST variations must lead to cancellations among different graphs to yield an overall BRST-invariant integrand. That is only possible if the BRST variation $Q N_{i}(\ell)$ cancels one of its propagators $P_{k, i}(\ell)$. Therefore, the superspace expression of $N_{i}(\ell)$ is constrained by the following requirement:

$$
\text { each term of } Q N_{i}(\ell) \text { must contain a factor of } P_{k, i}(\ell) \text {. }
$$

Otherwise the BRST variation of the integrand (3.1) would have a non-vanishing residue at the simultaneous pole $\prod_{k} P_{k, i}(\ell)$ and could not vanish.

We will use the following notation to distinguish between superspace integrands and integrated expressions,

$$
\begin{aligned}
\mathcal{A}_{n}^{2-\text { loop }} & =\int \frac{d^{D} \ell d^{D} r}{(2 \pi)^{2 D}}\left\langle\mathcal{A}_{n}^{2-\text { loop }}(\ell, r)\right\rangle \\
A^{2-\text { loop }}(1,2,3, \ldots, n) & =\int \frac{d^{D} \ell d^{D} r}{(2 \pi)^{2 D}}\left\langle A^{2-\text { loop }}(1,2,3, \ldots, n \mid \ell, r)\right\rangle,
\end{aligned}
$$

where $A^{2-\text { loop }}(1,2,3, \ldots, n)$ denotes the planar single-trace contribution of $\mathcal{A}_{n}^{2-\text { loop }}$ in the trace basis of $C_{i}$ [30]. In the following sections we will use the method outlined above to construct the SYM and supergravity five-point two-loop integrands. They lead to BRSTclosed integrated amplitudes once the freedom to shift or rename the integration variables $\ell, r$ is taken into account. ${ }^{3}$

\subsection{The SYM two-loop four-point amplitude}

Recall that the two-loop four-point amplitude of the pure spinor superstring [31-33] can be written in terms of a single kinematic factor

$$
\begin{aligned}
T_{1,2 \mid 3,4} & \equiv \frac{1}{64}\left(\lambda \gamma_{m n p q r} \lambda\right) F_{1}^{m n} F_{2}^{p q}\left[F_{3}^{r s}\left(\lambda \gamma_{s} W_{4}\right)+F_{4}^{r s}\left(\lambda \gamma_{s} W_{3}\right)\right]+(1,2 \leftrightarrow 3,4) \\
Q T_{1,2 \mid 3,4} & =0,
\end{aligned}
$$

\footnotetext{
${ }^{3}$ The diagrammatic bookkeeping of BRST variations in the appendix A automatically freezes this freedom by means of the automorphism symmetries of the different integrand topologies after the cancellation of propagators resulting from the BRST variation of the numerators.
} 


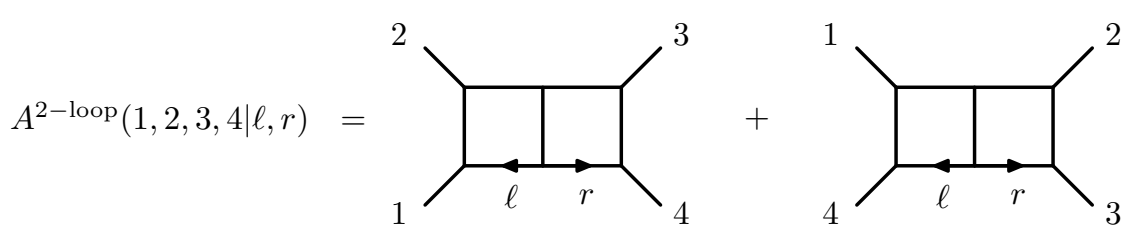

Figure 3. The planar integrand of the SYM two-loop four-point amplitude.

whose BRST invariance follows from the equations of motion (2.5) and the pure spinor constraint (2.10). Since superstring amplitude reduce to SYM amplitudes in field-theory limit, the most natural expression for the planar four-point two-loop integrand is given by

$$
\begin{aligned}
A^{2-\operatorname{loop}}(1,2,3,4 \mid \ell, r)= & \frac{T_{1,2 \mid 3,4}}{\ell^{2} r^{2}(\ell+r)^{2}\left(\ell-k_{1}\right)^{2}\left(\ell-k_{12}\right)^{2}\left(r-k_{4}\right)^{2}\left(r-k_{34}\right)^{2}} \\
& +\frac{T_{4,1 \mid 2,3}}{\ell^{2} r^{2}(\ell+r)^{2}\left(\ell-k_{4}\right)^{2}\left(\ell-k_{41}\right)^{2}\left(r-k_{3}\right)^{2}\left(r-k_{23}\right)^{2}} .
\end{aligned}
$$

The contributing double-box graphs are depicted in figure 3, and BRST invariance of its numerators is inherited from (3.4). Furthermore, it has been shown at the superspace level that [34]

$$
\left\langle T_{1,2 \mid 3,4}\right\rangle=s_{12}^{2} s_{23} A^{\text {tree }}(1,2,3,4),
$$

hence, the amplitude (3.5) agrees with the result of [35]. The Mandelstam invariants are

$$
s_{i j} \equiv\left(k_{i} \cdot k_{j}\right)=\frac{1}{2}\left(k_{i}+k_{j}\right)^{2}, \quad s_{i_{1} i_{2} \ldots i_{p}} \equiv \frac{1}{2}\left(k_{i_{1}}+k_{i_{2}}+\ldots+k_{i_{p}}\right)^{2} .
$$

A rigorous derivation of (3.5) as the field-theory limit of the two-loop open superstring amplitude [31] is expected to closely follow the closed-string discussion in [36].

\subsection{Five-point building blocks in pure spinor superspace}

By the aforementioned no-triangle property of maximal SYM [29], the two-loop five-point amplitude involves double-box and penta-box diagrams along with their non-planar counterparts. The numerators for the pentagon subdiagrams are known to depend linearly on the loop momentum [9], and therefore the five-point amplitude will require vector building blocks in addition to scalar building blocks.

For the scalar building block, one can use the multiparticle version of (3.4)

$$
T_{A, B \mid C, D} \equiv \frac{1}{64}\left(\lambda \gamma_{m n p q r} \lambda\right) F_{A}^{m n} F_{B}^{p q}\left[F_{C}^{r s}\left(\lambda \gamma_{s} W_{D}\right)+F_{D}^{r s}\left(\lambda \gamma_{s} W_{C}\right)\right]+(A, B \leftrightarrow C, D) .
$$

The symmetry properties of $T_{1,2 \mid 3,4}$ described in [31] do not depend on the single-particle nature of the superfields and directly carry over to (3.8),

$$
T_{A, B \mid C, D}=T_{B, A \mid C, D}=T_{C, D \mid A, B}, \quad T_{A, B \mid C, D}+T_{B, C \mid A, D}+T_{C, A \mid B, D}=0 .
$$

The latter follows from the gamma-matrix manipulation $\left(\lambda \gamma_{[m n p q r} \lambda\right)\left(\lambda \gamma_{s]}\right)_{\alpha}=0$. For five points, the BRST variation of (3.8) follows from multiparticle equations of motion (2.8),

$$
Q T_{12,3 \mid 4,5}=s_{12}\left(V_{1} T_{2,3 \mid 4,5}-V_{2} T_{1,3 \mid 4,5}\right),
$$

and the terms without a factor of $s_{12}$ drop out by virtue of the pure spinor constraint. 
In analogy to the one-loop vector building block of [18], the scalar two-loop building blocks (3.8) allow for a vector counterpart ${ }^{4}$ suitable to represent linear dependencies on $\ell$,

$$
T_{1,2,3 \mid 4,5}^{m} \equiv A_{1}^{m} T_{2,3 \mid 4,5}+A_{2}^{m} T_{1,3 \mid 4,5}+A_{3}^{m} T_{1,2 \mid 4,5}+W_{1,2,3 \mid 4,5}^{m} .
$$

The last summand $W_{1,2,3 \mid 4,5}^{m}$ is designed to cancel the term $\left(\lambda \gamma^{m} W_{1}\right)$ within $Q A_{1}^{m}$, i.e.

$$
Q W_{1,2,3 \mid 4,5}^{m}=-\left(\lambda \gamma^{m} W_{1}\right) T_{2,3 \mid 4,5}-\left(\lambda \gamma^{m} W_{2}\right) T_{1,3 \mid 4,5}-\left(\lambda \gamma^{m} W_{3}\right) T_{1,2 \mid 4,5}
$$

In a symmetrization convention where $W_{(1}^{\alpha} F_{2)}^{m n} \equiv W_{1}^{\alpha} F_{2}^{m n}+W_{2}^{\alpha} F_{1}^{m n}$, its explicit superfield representation is given by

$$
\begin{aligned}
W_{3,4,5 \mid 1,2}^{m} \equiv & \frac{1}{48}\left(\lambda \gamma_{p q} \gamma^{m} W_{(1}\right) F_{2)}^{p q}\left(\lambda \gamma_{r} W_{5}\right)\left(\lambda \gamma_{s} W_{(3}\right) F_{4)}^{r s} \\
& -\frac{1}{128}\left(\lambda \gamma^{m} W_{5}\right)\left(\lambda \gamma_{p q} \gamma^{r} W_{(1}\right) F_{2)}^{p q}\left(\lambda \gamma_{s t} \gamma_{r} W_{(3}\right) F_{4)}^{s t} \\
& +\frac{1}{96}\left(W_{3} \gamma^{m s t} W_{4}\right)\left(\lambda \gamma_{n p q r s} \lambda\right)\left(\lambda \gamma_{t} W_{5}\right) F_{1}^{n p} F_{2}^{q r}+(5 \leftrightarrow 3,4) .
\end{aligned}
$$

The BRST variation of $T_{1,2,3 \mid 4,5}^{m}$ in (3.11) then connects with the scalar counterpart (3.10),

$$
Q T_{1,2,3 \mid 4,5}^{m}=k_{1}^{m} V_{1} T_{2,3 \mid 4,5}+k_{2}^{m} V_{2} T_{1,3 \mid 4,5}+k_{3}^{m} V_{3} T_{1,2 \mid 4,5} .
$$

The symmetries (3.9) of the scalar building block and the form of $W_{1,2,3 \mid 4,5}^{m}$ in (3.13) imply

$$
T_{1,2,3 \mid 4,5}^{m}=T_{(1,2,3) \mid(4,5)}^{m}, \quad\left\langle T_{1,2,3 \mid 4,5}^{m}\right\rangle=\left\langle T_{3,4,5 \mid 1,2}^{m}+T_{2,4,5 \mid 1,3}^{m}+T_{1,4,5 \mid 2,3}^{m}\right\rangle .
$$

Note that the two-loop building blocks of this section are functionals of all the external labels, e.g. $T_{i, j, k \mid p, q}^{m}$ can be specialized to any permutation $(i, j, k, p, q)$ of $(1,2,3,4,5)$. This surpasses the constraints on superspace numerators at tree-level $[1,2]$ and oneloop [3] where individual external legs need to be globally associated with unintegrated vertices (2.13) or their multiparticle versions [23].

\section{The two-loop five-point amplitudes in SYM and supergravity}

In this section, we assemble the five-point two-loop amplitudes from the six topologies of cubic diagrams without triangles [9] depicted in figure 4.

\subsection{Color factors}

The color factors $C_{12345}^{(a)}, \ldots, C_{12345}^{(f)}$ associated to the diagrams of figure 4 encompass seven factors of $f^{a b c}$, the structure constants of the gauge group. Their contractions follow from dressing each cubic vertex ${ }^{5}$ of the diagram with a factor of $f^{a b c}$ and each internal line with

\footnotetext{
${ }^{4}$ The following definition can be generalized to multiparticle level $T_{1,2,3 \mid 4,5}^{m} \rightarrow T_{A, B, C \mid D, E}^{m}$ if the corresponding uplift of (3.13) is projected to the symmetries of (3.15).

${ }^{5}$ The color factors $C_{12345}^{(b)}, C_{12345}^{(e)}$ and $C_{12345}^{(f)}$ contain an extra minus sign w.r.t. [9] due to our choice of drawing graphs without crossing lines.
} 

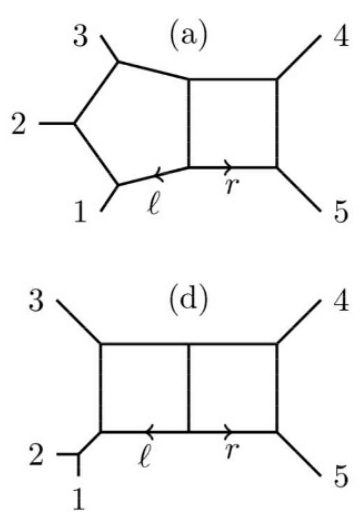

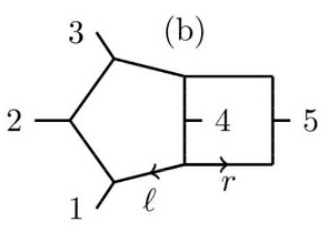

(e)

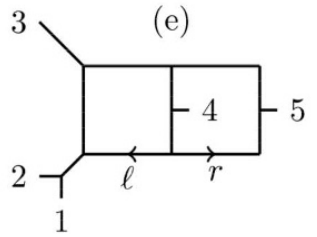

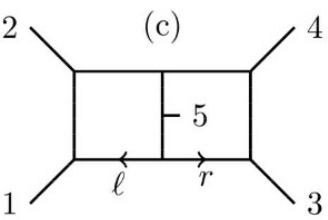

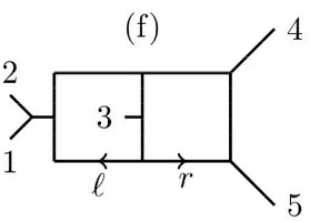

Figure 4. The six topologies of two-loop five-point diagrams whose color factors are given in (4.1). In a BCJ representation subject to (2.3), the numerators satisfy $N^{(b)}=-N^{(a)}$ as well as $N^{(f)}=$ $N^{(e)}=-N^{(d)}$, and the explicit superspace expressions for $N^{(d)}$ and $N^{(a)}$ are given in (4.2) and (4.3), respectively.

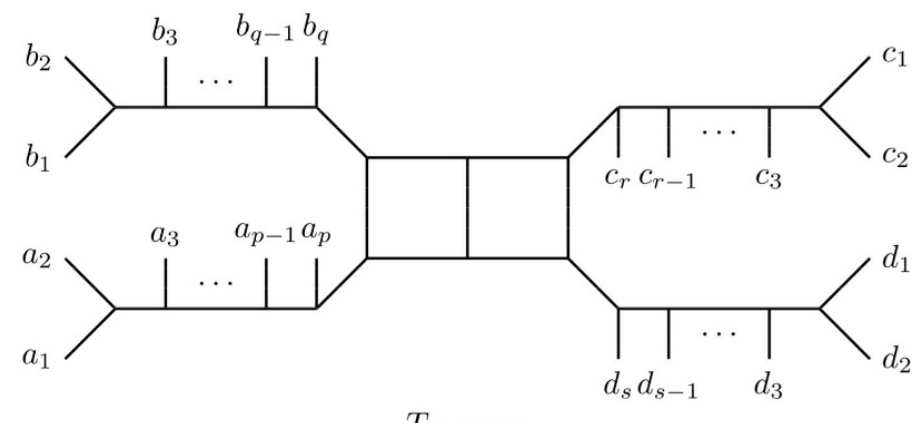

$T_{A, B \mid C, D}$

Figure 5. The numerator for the general massive double-box is given by $T_{A, B \mid C, D}$ in (3.8).

a Kronecker delta in the adjoint representation of the gauge group,

$$
\begin{array}{ll}
C_{12345}^{(a)}=f^{4 e c} f^{5 b e} f^{a 1 h} f^{b a d} f^{c d g} f^{g j 3} f^{h 2 j}, & C_{12345}^{(b)}=f^{4 d e} f^{b 5 c} f^{a 1 h} f^{c d g} f^{e b a} f^{g j 3} f^{h 2 j}, \\
C_{12345}^{(c)}=f^{1 a c} f^{2 h c} f^{a d g} f^{b 4 j} f^{e d 5} f^{g j 3} f^{h b e}, & C_{12345}^{(d)}=f^{4 e c} f^{5 b e} f^{a j h} f^{b a d} f^{c d g} f^{g j 3} f^{h 21}, \\
C_{12345}^{(e)}=f^{4 e c} f^{5 d b} f^{a j h} f^{b e a} f^{c d g} f^{g j 3} f^{h 12}, & C_{12345}^{(f)}=f^{12 c} f^{a d b} f^{b j 5} f^{c a g} f^{e 3 d} f^{g h e} f^{h 4 j} .
\end{array}
$$

Using the procedure described in [30] they can be translated into a trace basis leading to color structures of the form $N_{c}^{2} \operatorname{Tr}\left(t^{1} t^{2} t^{3} t^{4} t^{5}\right), \operatorname{Tr}\left(t^{1} t^{2} t^{3} t^{4} t^{5}\right), N_{c} \operatorname{Tr}\left(t^{4} t^{5}\right) \operatorname{Tr}\left(t^{1} t^{2} t^{3}\right)$, $\operatorname{Tr}\left(t^{4}\right) \operatorname{Tr}\left(t^{5}\right) \operatorname{Tr}\left(t^{1} t^{2} t^{3}\right)$ and $N_{c} \operatorname{Tr}\left(t^{5}\right) \operatorname{Tr}\left(t^{1} t^{2} t^{3} t^{4}\right)$. The gauge group is left completely general at this point such that its generators $t^{i}$ are not necessarily traceless. The number of colors $N_{c}$ stems from the trace of the identity matrix.

\subsection{Planar cubic graphs and superspace numerators}

The BRST cohomology principle (3.2) suggests the scalar multiparticle building block $T_{A, B \mid C, D}$ in (3.8) to furnish a massive double-box numerator, see figure 5. This conjectural identification is based on the fact that all the $\ell$-independent propagators in figure 5 
are canceled by some summand of $Q T_{A, B \mid C, D}$, see e.g. (3.10). Moreover, the symmetry $T_{A, B \mid C, D}=T_{A, B \mid D, C}$ is compatible with the no-triangle property and the kinematic Jacobi identity:

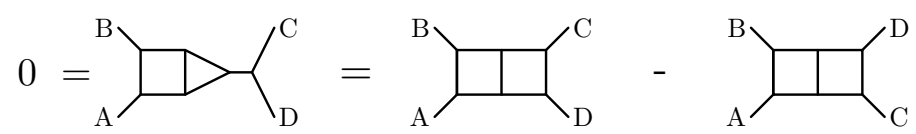

The dictionary in figure 5 implies that the planar five-point double-box in $4(\mathrm{~d})$ is represented by the numerator

$$
N_{12,3 \mid 4,5}^{(d)} \equiv T_{12,3 \mid 4,5}
$$

The scalar and vector BRST transformations in (3.10) and (3.14) allow to construct a candidate for the penta-box numerator of figure $4(\mathrm{a})$ as

$$
N_{1,2,3 \mid 4,5}^{(a)}(\ell) \equiv \frac{1}{2}\left(\ell_{m}+\ell_{m}-k_{m}^{123}\right) T_{1,2,3 \mid 4,5}^{m}+\frac{1}{2}\left(T_{12,3 \mid 4,5}+T_{13,2 \mid 4,5}+T_{23,1 \mid 4,5}\right) .
$$

This expression is designed from the cohomology principle (3.2) since $\ell$-dependent propagators are canceled under the BRST variation,

$$
\begin{gathered}
Q N_{1,2,3 \mid 4,5}^{(a)}(\ell)=\frac{1}{2}\left(V_{1} T_{2,3 \mid 4,5}\left[\ell^{2}-\left(\ell-k_{1}\right)^{2}\right]+V_{2} T_{1,3 \mid 4,5}\left[\left(\ell-k_{1}\right)^{2}-\left(\ell-k_{12}\right)^{2}\right]\right. \\
\left.+V_{3} T_{1,2 \mid 4,5}\left[\left(\ell-k_{12}\right)^{2}-\left(\ell-k_{123}\right)^{2}\right]\right) .
\end{gathered}
$$

Both the composition (4.3) from scalar and vector superfields and the form of the BRST variation (4.4) resemble the one-loop pentagon numerator, see (4.5) and (4.6) of [3].

As illustrated in figure 6, the numerator in (4.3) depends on the averaged loop momentum $\ell_{m}+\left(\ell_{m}-k_{m}^{123}\right)$ from the two terminal edges of the pentagonic worldline segment. This makes sure that the numerator inherits the reflection symmetry of the cubic diagram in figure 4(a) [9],

$$
N_{1,2,3 \mid 4,5}^{(a)}(\ell)=-N_{3,2,1 \mid 5,4}^{(a)}\left(k_{123}-\ell\right) .
$$

Also, the symmetry of $N_{1,2,3 \mid 4,5}^{(a)}(\ell)$ in 4,5 is compatible with the no-triangle property:

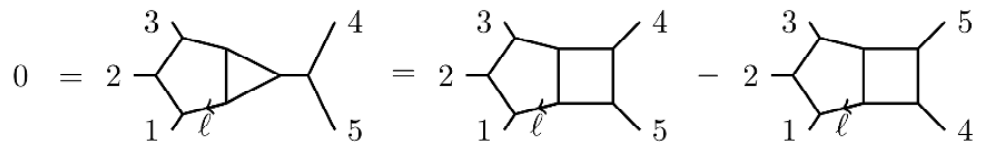

Finally it is easy to show the kinematic Jacobi identity [9]

$$
N_{12,3 \mid 4,5}^{(d)}=N_{1,2,3 \mid 4,5}^{(a)}(\ell)-N_{2,1,3 \mid 4,5}^{(a)}(\ell)
$$

which interlocks antisymmetrized penta-boxes with double-boxes.

\subsection{Planar two-loop amplitudes in SYM}

The numerators (3.8) and (4.3) for planar double-box and penta-box diagrams are summarized in figure 6 and satisfy the BRST cohomology principle (3.2). Hence, we propose 

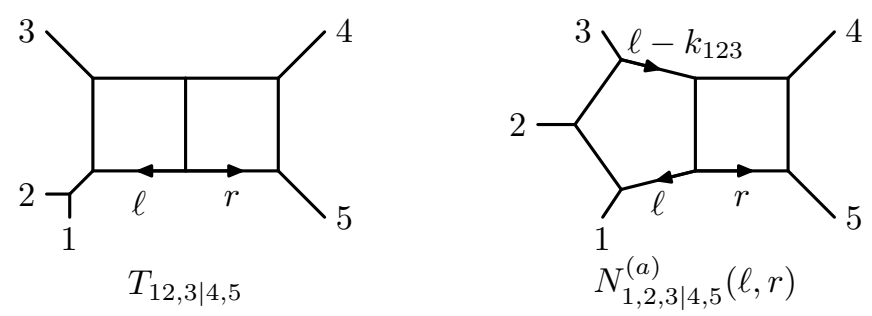

Figure 6. The mapping between the double-box and penta-box graphs and superspace numerators. The vertical bar in the notation ... . . separates the two worldline segments.

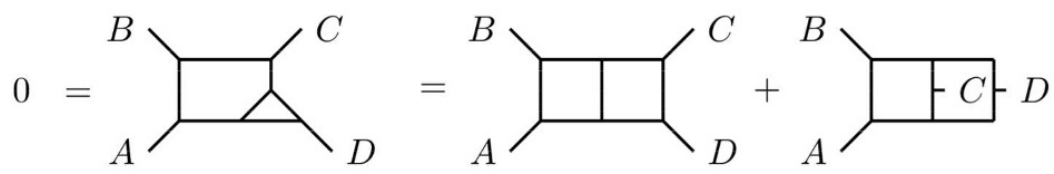

Figure 7. The derivation of $N_{12,3 \mid 4,5}^{(e)}=N_{12,3 \mid 4,5}^{(f)}=-N_{12,3 \mid 4,5}^{(d)}$ from a kinematic Jacobi identity and the no-triangle property. A similar analysis leads to $N_{1,2,3 \mid 4,5}^{(b)}=-N_{1,2,3 \mid 4,5}^{(a)}$.

the following single-trace five-point integrand for (3.3):

$$
\begin{aligned}
A^{2-\operatorname{loop}}(1,2,3,4,5 \mid \ell, r)= & \frac{1}{\ell^{2} r^{2}(\ell+r)^{2}}\left[\frac{N_{1,2,3 \mid 4,5}^{(a)}(\ell)}{\left(\ell-k_{1}\right)^{2}\left(\ell-k_{12}\right)^{2}\left(\ell-k_{123}\right)^{2}\left(r-k_{5}\right)^{2}\left(r-k_{45}\right)^{2}}\right. \\
& +\frac{N_{12,3 \mid 4,5}^{(d)}}{k_{12}^{2}\left(\ell-k_{12}\right)^{2}\left(\ell-k_{123}\right)^{2}\left(r-k_{5}\right)^{2}\left(r-k_{45}\right)^{2}} \\
& \left.+\frac{N_{5,12 \mid 3,4}^{(d)}}{k_{12}^{2}\left(\ell-k_{5}\right)^{2}\left(\ell-k_{512}\right)^{2}\left(r-k_{4}\right)^{2}\left(r-k_{34}\right)^{2}}+\operatorname{cyc}(1,2,3,4,5)\right] .
\end{aligned}
$$

Given that cyc $(1,2,3,4,5)$ instructs to add the four cyclic images of $(1,2,3,4,5)$, the expression in (4.7) is manifestly cyclic, and its BRST invariance is easily checked with a diagrammatic bookkeeping of the associated integrals, see the appendix A for more details.

\subsection{Non-planar cubic graphs and superspace numerators}

In contrast with the one-loop level [29], single-trace subamplitudes at two-loops are no longer sufficient to infer the kinematic structure associated with all color tensors. Candidate expressions for the non-planar diagrams in term of the planar numerators can be obtained from the BCJ duality and have been worked out for the five-point two-loop case in [9]. Non-planar double-boxes follow from the no-triangle property and the kinematic Jacobi relation shown in figure 7 ,

$$
N_{12,3 \mid 4,5}^{(e)}=N_{12,3 \mid 4,5}^{(f)}=-N_{12,3 \mid 4,5}^{(d)} .
$$


The numerators for non-planar penta-box diagrams are obtained from a sequence of similar BCJ moves and given by [9]

$$
\begin{aligned}
N_{1,2,3 \mid 4,5}^{(b)}(\ell)= & -N_{1,2,3 \mid 4,5}^{(a)}(\ell) \\
N_{1,2|4,3| 5}^{(c)}(\ell, r)= & N_{1,2,5 \mid 3,4}^{(a)}(\ell)+N_{3,4,5 \mid 1,2}^{(a)}(r) \\
= & \frac{1}{2}\left(\ell_{m}+\ell_{m}-k_{m}^{125}\right) T_{1,2,5 \mid 3,4}^{m}+\frac{1}{2}\left(r_{m}+r_{m}-k_{m}^{345}\right) T_{3,4,5 \mid 1,2}^{m} \\
& +\frac{1}{2}\left(T_{12,5 \mid 3,4}+T_{25,1 \mid 3,4}+T_{15,2 \mid 3,4}+T_{34,5 \mid 1,2}+T_{35,4 \mid 1,2}+T_{45,3 \mid 1,2}\right) .
\end{aligned}
$$

As a non-trivial consistency condition on the superspace numerator (4.10), one can check its compatibility with the BRST principle (3.2) through the BRST variation

$$
\begin{aligned}
2 Q N_{1,2|4,3| 5}^{(c)}(\ell, r)= & V_{5} T_{1,2 \mid 3,4}\left[\left(\ell+r+k_{5}\right)^{2}-(\ell+r)^{2}\right] \\
& +V_{1} T_{2,5 \mid 3,4}\left[\ell^{2}-\left(\ell-k_{1}\right)^{2}\right]+V_{2} T_{1,5 \mid 3,4}\left[\left(\ell-k_{1}\right)^{2}-\left(\ell-k_{12}\right)^{2}\right] \\
& +V_{3} T_{4,5 \mid 1,2}\left[r^{2}-\left(r-k_{3}\right)^{2}\right]+V_{4} T_{3,5 \mid 1,2}\left[\left(r-k_{3}\right)^{2}-\left(r-k_{34}\right)^{2}\right] .
\end{aligned}
$$

Moreover, (4.10) satisfies the required self-symmetries [9]. For example, a rotation by $180^{\circ}$ (with a simultaneous flip of the vertex next to particle 5) maps the diagram to itself up to relabellings:

$$
N_{1,2|4,3| 5}^{(c)}(\ell, r)=-N_{4,3|1,2| 5}^{(c)}\left(k_{34}-r, k_{12}-\ell\right)
$$

This is respected by the expression in (4.10) provided that $^{6}$

$$
k_{m}^{5}\left\langle T_{3,4,5 \mid 1,2}^{m}+T_{1,2,5 \mid 3,4}^{m}\right\rangle=\left\langle T_{15,2 \mid 3,4}+T_{25,1 \mid 3,4}+T_{35,4 \mid 1,2}+T_{45,3 \mid 1,2}\right\rangle .
$$

Clearly, the difference of the superfields on the two sides is BRST closed, and a component evaluation confirms (4.13). In fact, any BRST-closed and local expression obtained from permutation of $T_{12,3 \mid 4,5}, k_{m}^{1} T_{1,2,3 \mid 4,5}^{m}$ or $k_{m}^{4} T_{1,2,3 \mid 4,5}^{m}$ is checked to have vanishing components, i.e. any element in the BRST cohomology formed from the above two-loop building blocks requires kinematic poles.

The above numerators $N^{(a)}, \ldots, N^{(f)}$ can be mapped to the four-dimensional counterparts of [9] by identifying each permutation of $T_{12,3 \mid 4,5}$ and $T_{1,2,3 \mid 4,5}^{m}$ with certain spinor helicity expressions specified in the appendix B. This mapping is only of formal nature and does not result from a (straightforwardly applicable) dimensional reduction and specialization to four-dimensional spinor-helicity polarizations: the ten-dimensional superspace numerators are local expressions of polarizations and momenta while the spinor-helicity expressions of [9] contain highly non-local inverse Gram determinant factors.

\footnotetext{
${ }^{6}$ Surprisingly, (4.13) is also the condition for BRST invariance of the corresponding closed string fivepoint amplitude at two loops.
} 


\subsection{Non-planar two-loop amplitudes in SYM}

The color-dressed SYM amplitude follows from assembling the six topologies of cubic diagrams depicted in figure 4 [9]. In a shorthand notation for the propagators

$$
\begin{aligned}
I_{1,2,3,4,5}^{(a)} & \equiv \frac{1}{\ell^{2} r^{2}(\ell+r)^{2}\left(\ell-k_{1}\right)^{2}\left(\ell-k_{12}\right)^{2}\left(\ell-k_{123}\right)^{2}\left(r-k_{5}\right)^{2}\left(r-k_{45}\right)^{2}} \\
I_{1,2,3,4,5}^{(b)} & \equiv \frac{1}{\ell^{2} r^{2}(\ell+r)^{2}\left(\ell-k_{1}\right)^{2}\left(\ell-k_{12}\right)^{2}\left(\ell-k_{123}\right)^{2}\left(r-k_{5}\right)^{2}\left(\ell+r+k_{4}\right)^{2}} \\
I_{1,2,3,4,5}^{(c)} & \equiv \frac{1}{\ell^{2} r^{2}(\ell+r)^{2}\left(\ell-k_{1}\right)^{2}\left(\ell-k_{12}\right)^{2}\left(r-k_{3}\right)^{2}\left(r-k_{34}\right)^{2}\left(\ell+r+k_{5}\right)^{2}} \\
I_{1,2,3,4,5}^{(d)} & \equiv \frac{1}{k_{12}^{2} \ell^{2} r^{2}(\ell+r)^{2}\left(\ell-k_{12}\right)^{2}\left(\ell-k_{123}\right)^{2}\left(r-k_{5}\right)^{2}\left(r-k_{45}\right)^{2}} \\
I_{1,2,3,4,5}^{(e)} & \equiv \frac{1}{k_{12}^{2} \ell^{2} r^{2}(\ell+r)^{2}\left(\ell-k_{12}\right)^{2}\left(\ell-k_{123}\right)^{2}\left(r-k_{5}\right)^{2}\left(r+\ell+k_{4}\right)^{2}} \\
I_{1,2,3,4,5}^{(f)} & \equiv \frac{1}{k_{12}^{2} \ell^{2} r^{2}(\ell+r)^{2}\left(\ell-k_{12}\right)^{2}\left(\ell+r+k_{3}\right)^{2}\left(r-k_{5}\right)^{2}\left(r-k_{45}\right)^{2}},
\end{aligned}
$$

we have

$$
\begin{aligned}
\mathcal{A}_{5}^{2-\text { loop }}(\ell, r)= & \frac{1}{2} N_{1,2,3 \mid 4,5}^{(a)}(\ell) C_{12345}^{(a)} I_{1,2,3,4,5}^{(a)}+\frac{1}{4} N_{1,2,3 \mid 4,5}^{(b)}(\ell) C_{12345}^{(b)} I_{1,2,3,4,5}^{(b)} \\
& +\frac{1}{4} N_{1,2|4,3| 5}^{(c)}(\ell, r) C_{12345}^{(c)} I_{1,2,3,4,5}^{(c)}+\frac{1}{2} N_{12,3 \mid 4,5}^{(d)} C_{12345}^{(d)} I_{1,2,3,4,5}^{(d)} \\
& +\frac{1}{4} N_{12,3 \mid 4,5}^{(e)} C_{12345}^{(e)} I_{1,2,3,4,5}^{(e)}+\frac{1}{4} N_{12,3 \mid 4,5}^{(f)} C_{12345}^{(f)} I_{1,2,3,4,5}^{(f)}+\operatorname{sym}(1,2,3,4,5) .
\end{aligned}
$$

The notation $\operatorname{sym}(1,2,3,4,5)$ instructs to sum over all the 120 permutations of $(1,2,3,4,5)$, and the symmetry factors $\frac{1}{2}$ and $\frac{1}{4}$ avoid overcounting in the permutation sum [9]. The coefficients of all inequivalent trace configurations in (4.15) following from (4.1) are checked to be independently BRST invariant, i.e. the complete color-dressed amplitude (4.15) is BRST closed. More details can be found in the appendix A.

\subsection{Two-loop amplitudes in supergravity}

Since the color-dressed two-loop five-point SYM amplitude (4.15) is written in terms of numerators which satisfy the BCJ color-kinematics duality, the corresponding supergravity integrand is readily obtained by squaring its numerators [10]

$$
\begin{aligned}
\mathcal{M}_{5}^{2-\text { loop }}(\ell, r)= & \frac{1}{2}\left|N_{1,2,3 \mid 4,5}^{(a)}(\ell)\right|^{2} I_{1,2,3,4,5}^{(a)}+\frac{1}{4}\left|N_{1,2,3 \mid 4,5}^{(b)}(\ell)\right|^{2} I_{1,2,3,4,5}^{(b)} \\
& +\frac{1}{4}\left|N_{1,2|4,3| 5}^{(c)}(\ell, r)\right|^{2} I_{1,2,3,4,5}^{(c)}+\frac{1}{2}\left|N_{12,3 \mid 4,5}^{(d)}\right|^{2} I_{1,2,3,4,5}^{(d)} \\
& +\frac{1}{4}\left|N_{12,3 \mid 4,5}^{(e)}\right|^{2} I_{1,2,3,4,5}^{(e)}+\frac{1}{4}\left|N_{12,3 \mid 4,5}^{(f)}\right|^{2} I_{1,2,3,4,5}^{(f)}+\operatorname{sym}(1,2,3,4,5),
\end{aligned}
$$

where $\left|N^{(i)}\right|^{2}$ is a shorthand for $N^{(i)} \tilde{N}^{(i)}$ with $i=a, b, c, d, e, f$. The amplitude (4.16) describes type IIB or type IIA supergravity if the $\mathrm{SO}(10)$ Weyl spinors within the "rightmoving" numerators $\tilde{N}^{(i)}$ have the same or opposite chirality as compared to the "leftmovers" $N^{(i)}$. BRST invariance with respect to the left-movers $N^{(i)}$ is inherited from the 
SYM amplitude (4.15) since the accompanying right-moving numerators $\tilde{N}^{(i)}$ satisfy all the Jacobi identities of the color factors $C^{(i)}$. The argument extends to the right-moving BRST variation upon exchange of $N^{(i)}$ and $\tilde{N}^{(i)}$.

\section{UV divergences}

In this section, we compute the UV divergences of the above two-loop five-point amplitudes in SYM and supergravity and rewrite the kinematic factors in terms of SYM tree amplitudes. They are confirmed to match the low-energy limit of the corresponding superstring amplitudes. For completeness and comparison across different loop-orders, we provide a dimension-agnostic representation of the one-loop five-point UV divergences in the appendix C.

\subsection{Two-loop UV divergences in SYM}

In the above BCJ representation of the two-loop five-point SYM amplitude, the kinematic numerators are at most linear in the loop momentum. Together with the no-triangle property, this implies that the double-box diagrams dominate in the UV regime of large $\ell^{2}$. In an expansion around the two-loop critical dimension $D=7-2 \epsilon$ [37], the planar and non-planar box graphs contribute as follows in the UV [35, 38],

$$
\begin{aligned}
\int \frac{d^{7-2 \epsilon} \ell d^{7-2 \epsilon} r(2 \pi)^{4 \epsilon-14}}{\ell^{2} r^{2}(\ell+r)^{2}\left(\ell-k_{1}\right)^{2}\left(\ell-k_{12}\right)^{2}\left(r-k_{4}\right)^{2}\left(r-k_{34}\right)^{2}} & =-\frac{\pi+\mathcal{O}(\epsilon)}{20(4 \pi)^{7} \epsilon} \equiv V^{(\mathrm{P})} \\
\int \frac{d^{7-2 \epsilon} \ell d^{7-2 \epsilon} r(2 \pi)^{4 \epsilon-14}}{\ell^{2} r^{2}(\ell+r)^{2}\left(\ell+r+k_{3}\right)^{2}\left(\ell-k_{1}\right)^{2}\left(\ell-k_{12}\right)^{2}\left(r-k_{4}\right)^{2}} & =-\frac{\pi+\mathcal{O}(\epsilon)}{30(4 \pi)^{7} \epsilon} \equiv V^{(\mathrm{NP})},
\end{aligned}
$$

whereas penta-box diagrams are regular in the dimensional regularization parameter $\epsilon$.

The superspace representations for the single-trace subamplitudes (3.5) and (4.7) yield the following UV divergence in the critical dimension $D=7$ :

$$
\begin{aligned}
\left.A^{2-\text { loop }}(1,2,3,4)\right|_{\mathrm{UV}} & =V^{(\mathrm{P})}\left\langle T_{1,2 \mid 3,4}+T_{4,1 \mid 2,3}\right\rangle=-V^{(\mathrm{P})}\left\langle T_{1,3 \mid 2,4}\right\rangle \\
\left.A^{2-\operatorname{loop}}(1,2,3,4,5)\right|_{\mathrm{UV}} & =-V^{(\mathrm{P})}\left\langle\frac{T_{12,4 \mid 3,5}}{s_{12}}+\frac{T_{23,5 \mid 1,4}}{s_{23}}+\frac{T_{1,34 \mid 2,5}}{s_{34}}+\frac{T_{2,45 \mid 1,3}}{s_{45}}+\frac{T_{51,3 \mid 2,4}}{s_{51}}\right\rangle
\end{aligned}
$$

The associated counterterm is the supersymmetrized operator $\operatorname{Tr}\left(D^{2} F^{4}+F^{5}\right)$ which also finds appearance in the tree-level effective action of the open superstring at order $\alpha^{\prime 3}[39,40]$. Superspace arguments of [34] and a component evaluation via [25] confirm that the kinematic factors in (5.3) are related to SYM tree amplitudes via

$$
\begin{gathered}
\left.A^{2-\operatorname{loop}}(1,2,3,4)\right|_{\mathrm{UV}}=V^{(\mathrm{P})} s_{12} s_{13} s_{23} A^{\text {tree }}(1,2,3,4) \\
\left.\left(\begin{array}{l}
A^{2-\operatorname{loop}}(1,2,3,4,5) \\
A^{2-\operatorname{loop}}(1,3,2,4,5)
\end{array}\right)\right|_{\mathrm{UV}}=V^{(\mathrm{P})} M_{3} \cdot\left(\begin{array}{l}
A^{\text {tree }}(1,2,3,4,5) \\
A^{\text {tree }}(1,3,2,4,5)
\end{array}\right) .
\end{gathered}
$$


The $2 \times 2$ matrix $M_{3}$ has been introduced in [41] to describe the momentum dependence of the $\alpha^{\prime}$-corrections in open superstring tree-amplitudes [42, 43] (see also [44, 45]). Its entries are given by

$$
M_{3} \equiv\left(\begin{array}{ll}
m_{11} & m_{12} \\
m_{21} & m_{22}
\end{array}\right), \quad \begin{aligned}
& m_{12}=-s_{13} s_{24}\left(s_{1}+s_{2}+s_{3}+s_{4}+s_{5}\right) \\
& m_{11}=s_{3}\left[-s_{1}\left(s_{1}+2 s_{2}+s_{3}\right)+s_{3} s_{4}+s_{4}^{2}\right]+s_{1} s_{5}\left(s_{1}+s_{5}\right)
\end{aligned}
$$

with $m_{21}=\left.m_{12}\right|_{2 \leftrightarrow 3}$ and $m_{22}=\left.m_{11}\right|_{2 \leftrightarrow 3}$ as well as $s_{i} \equiv s_{i, i+1}$ subject to $s_{5}=s_{15}$. As a main virtue of writing the UV divergence (5.4) in terms of $A^{\text {tree }}(\ldots)$, it is agnostic to the choice of kinematic variables and can be adapted to any spacetime dimension by choosing the appropriate representation of the SYM tree.

Upon combination with the non-planar sector, the color-dressed amplitude (4.15) for traceless gauge group generators $\operatorname{Tr}\left(t^{i}\right)=0$ yields the UV divergence

$$
\begin{aligned}
\left.\mathcal{A}_{5}^{2-\text { loop }}\right|_{\mathrm{UV}}= & {\left[V^{(\mathrm{P})} N_{c}^{2}+12\left(V^{(\mathrm{P})}+V^{(\mathrm{NP})}\right)\right] } \\
& \times\left\{\left(\begin{array}{c}
\operatorname{Tr}\left(t^{1} t^{2} t^{3} t^{4} t^{5}\right) \\
\operatorname{Tr}\left(t^{1} t^{3} t^{2} t^{4} t^{5}\right)
\end{array}\right)^{T} \cdot M_{3} \cdot\left(\begin{array}{c}
A^{\text {tree }}(1,2,3,4,5) \\
A^{\text {tree }}(1,3,2,4,5)
\end{array}\right)+(2,3 \mid 2,3,4,5)\right\} \\
& -12 N_{c}\left(V^{(\mathrm{P})}+V^{(\mathrm{NP})}\right)\left\{\operatorname{Tr}\left(t^{1} t^{2} t^{3}\right) \operatorname{Tr}\left(t^{4} t^{5}\right)\right. \\
& \left.\times s_{45}^{2}\left[s_{24} A^{\text {tree }}(1,3,2,4,5)-s_{34} A^{\text {tree }}(1,2,3,4,5)\right]+(4,5 \mid 1,2,3,4,5)\right\}
\end{aligned}
$$

after expanding the color factors $C_{i}$ in a trace basis. The notation $\left(a_{1}, a_{2} \mid a_{1}, a_{2}, \ldots, a_{n}\right)$ instructs to sum over all possible ways to choose two elements out of the set $\left(a_{1}, a_{2}, \ldots, a_{n}\right)$, for a total of $\left(\begin{array}{l}n \\ 2\end{array}\right)$ terms. The coefficients of the multitrace color structure $\operatorname{Tr}\left(t^{1} t^{2} t^{3}\right) \operatorname{Tr}\left(t^{4} t^{5}\right)$ stem from the kinematic factor

$$
\left\langle\frac{T_{12,3 \mid 4,5}}{s_{12}}+\frac{T_{23,1 \mid 4,5}}{s_{23}}+\frac{T_{31,2 \mid 4,5}}{s_{13}}\right\rangle=s_{45}^{2}\left[s_{24} A^{\text {tree }}(1,3,2,4,5)-s_{34} A^{\text {tree }}(1,2,3,4,5)\right],
$$

which reduces to the four-dimensional expression $s_{45}\left(\frac{\gamma_{12}}{s_{12}}+\frac{\gamma_{23}}{s_{23}}+\frac{\gamma_{31}}{s_{31}}\right)$ in [9] under the mapping (B.1). Similarly, the single-trace kinematic factor in (5.3) is mapped to the spinor helicity expression $\beta_{12345}+\frac{\gamma_{12}}{s_{12}}\left(s_{35}-2 s_{12}\right)+\operatorname{cyc}(1,2,3,4,5)$ from [9] under (B.1).

\subsection{Two-loop UV divergences in supergravity}

According to their BCJ construction, the penta-box numerators in the supergravity amplitude (4.16) involve up to two powers of loop momentum whereas double-box numerators remain independent on $\ell$. Accordingly, the leading UV contributions in $D=7-2 \epsilon$ dimensions are given by

$$
\begin{gathered}
\left.\mathcal{M}_{4}^{2-\text { loop }}\right|_{\mathrm{UV}}=2\left(V^{(\mathrm{P})}+V^{(\mathrm{NP})}\right)\left\{\left|\left\langle T_{1,2 \mid 3,4}\right\rangle\right|^{2}+\left|\left\langle T_{1,3 \mid 2,4}\right\rangle\right|^{2}+\left|\left\langle T_{1,4 \mid 2,3}\right\rangle\right|^{2}\right\} \\
\left.\mathcal{M}_{5}^{2-\text { loop }}\right|_{\mathrm{UV}}=2\left(V^{(\mathrm{P})}+V^{(\mathrm{NP})}\right)\left\{\frac{\left|\left\langle T_{12,3 \mid 4,5}\right\rangle\right|^{2}}{s_{12}}+\frac{\left|\left\langle T_{12,4 \mid 3,5}\right\rangle\right|^{2}}{s_{12}}+\frac{\left|\left\langle T_{12,5 \mid 3,4}\right\rangle\right|^{2}}{s_{12}}\right. \\
\left.+\left|\left\langle T_{3,4,5 \mid 1,2}^{m}\right\rangle\right|^{2}+(1,2 \mid 1,2,3,4,5)\right\} .
\end{gathered}
$$


As a consistency check of the proposed supergravity amplitude (4.16), the above UV divergences have to agree with the low-energy limits of the two-loop closed superstring amplitudes [13]. Indeed, the kinematic factors in (5.8) emerge in the low-energy limits at four-points [31] and five-points ${ }^{7}$ [14]. The polarization dependence can be written in terms of SYM tree subamplitudes: at four points, the superspace arguments of [34] imply that

$$
\left|\left\langle T_{1,2 \mid 3,4}\right\rangle\right|^{2}+\left|\left\langle T_{1,3 \mid 2,4}\right\rangle\right|^{2}+\left|\left\langle T_{1,4 \mid 2,3}\right\rangle\right|^{2}=\left(s_{12}^{2}+s_{13}^{2}+s_{23}^{2}\right)\left|s_{12} s_{23} A^{\text {tree }}(1,2,3,4)\right|^{2},
$$

and a ten-dimensional type IIB component evaluation at five points yields [14]

$$
\begin{aligned}
& \frac{\left|\left\langle T_{12,3 \mid 4,5}\right\rangle\right|^{2}}{s_{12}}+\frac{\left|\left\langle T_{12,4 \mid 3,5}\right\rangle\right|^{2}}{s_{12}}+\frac{\left|\left\langle T_{12,5 \mid 3,4}\right\rangle\right|^{2}}{s_{12}}+\left|\left\langle T_{3,4,5 \mid 1,2}^{m}\right\rangle\right|^{2}+\left.(1,2 \mid 1,2,3,4,5)\right|_{\mathrm{IIB}} ^{D=10} \\
& =2\left(\begin{array}{c}
\tilde{A}^{\text {tree }}(1,2,3,5,4) \\
\tilde{A}^{\text {tree }}(1,3,2,5,4)
\end{array}\right)^{T} \cdot S_{0} \cdot M_{5} \cdot\left(\begin{array}{l}
A^{\text {tree }}(1,2,3,4,5) \\
A^{\text {tree }}(1,3,2,4,5)
\end{array}\right) \times\left\{\begin{array}{rcc}
1 & : & h^{5} \\
-\frac{3}{5} & : & \phi h^{4}
\end{array} .\right.
\end{aligned}
$$

The $2 \times 2$ matrix $M_{5}$ with entries of order $s_{i j}^{5}$ has been introduced in [41] to describe the $\left(\alpha^{\prime}\right)^{5}$-correction to the open five-point superstring tree-level amplitude [42, 43] and can be downloaded from the website [46]. The matrix

$$
S_{0} \equiv\left(\begin{array}{cc}
s_{12}\left(s_{13}+s_{23}\right) & s_{12} s_{13} \\
s_{12} s_{13} & s_{13}\left(s_{12}+s_{23}\right)
\end{array}\right)
$$

captures the Mandelstam invariants in the field-theory limit of the KLT relations [47]. The shorthands $h^{5}$ and $\phi h^{4}$ in (5.11) refer to type IIB components with zero and two units of R-symmetry charge such as five gravitons $h^{5}$ or four gravitons and one (axio-)dilaton $\phi h^{4}$, respectively.

\subsection{UV divergence and R-symmetry}

As seen in (5.9) and (5.11), the UV divergence of the supergravity two-loop five-point amplitude is given by the same superspace expression that arises in the low-energy limit of the corresponding closed-string amplitude computed in [14]. Furthermore, the string amplitude for R-symmetry violating states such as $\phi h^{4}$ does not vanish; its characteristic coefficient $-3 / 5$ in (5.11) agrees with expectations from S-duality considerations for the type IIB string $[14,16,17]$. These facts give rise to worry that the two-loop UV divergence in supergravity might violate the R-symmetry as well.

However, that is not the case. ${ }^{8}$ To see this, note that the two-loop UV divergence of supergravity occurs in the critical dimension $D=7$ whereas the string $\phi h^{4}$ amplitude (5.11) is computed in $D=10$. Furthermore, recall that the graviton polarization $h_{m n}$ is the traceless part of $e_{(m} \tilde{e}_{n)}$ while the dilaton wavefunction $\left(\delta_{m n}-k_{m} \bar{k}_{n}-k_{n} \bar{k}_{m}\right) \phi$ covers the trace part with respect to the little group whose reference momentum $\bar{k}_{m}$ satisfies $\bar{k} \cdot \bar{k}=0$

\footnotetext{
${ }^{7}$ The five-point superstring computation in [14] leads to a different representation of $T_{A, B \mid C, D}$ and $T_{1,2,3 \mid 4,5}^{m}$ in terms of the non-minimal pure spinor variables [28]. However, BRST-invariant expressions do not depend on the representation of their composing building block.

${ }^{8}$ We thank John-Joseph Carrasco and Henrik Johansson for helpful email correspondence on this point.
} 
and $k \cdot \bar{k}=1$ [48]. Care must be taken when amplitudes involving dilatons are computed in general dimensions $D$, since the dimensional reduction of the little group trace yields

$$
e \cdot \tilde{e}=(D-2) \phi
$$

Note that the four-dimensional dilaton state is tied to R-symmetry anomalies in $D=4$ supergravities with $\mathcal{N} \leq 4$ supersymmetry, see e.g. [49].

In fact, using the component form of the building blocks $T_{12,3 \mid 4,5}$ and $T_{1,2,3 \mid 4,5}^{m}$ available to download in [11] one can check that the kinematic factor (5.11) in $D$ dimensions becomes ${ }^{9}$

$$
\begin{aligned}
& \frac{\left|\left\langle T_{12,3 \mid 4,5}\right\rangle\right|^{2}}{s_{12}}+\frac{\left|\left\langle T_{12,4 \mid 3,5}\right\rangle\right|^{2}}{s_{12}}+\frac{\left|\left\langle T_{12,5 \mid 3,4}\right\rangle\right|^{2}}{s_{12}}+\left|\left\langle T_{3,4,5 \mid 1,2}^{m}\right\rangle\right|^{2}+\left.(1,2 \mid 1,2,3,4,5)\right|_{\text {IIB }} ^{D} \\
& =2\left(\begin{array}{c}
\tilde{A}^{\text {tree }}(1,2,3,5,4) \\
\tilde{A}^{\text {tree }}(1,3,2,5,4)
\end{array}\right)^{T} \cdot S_{0} \cdot M_{5} \cdot\left(\begin{array}{l}
A^{\text {tree }}(1,2,3,4,5) \\
A^{\text {tree }}(1,3,2,4,5)
\end{array}\right) \times\left\{\begin{array}{ccc}
1 & : & h^{5} \\
\frac{(7-D)}{5} & : & \phi h^{4}
\end{array}\right.
\end{aligned}
$$

Therefore, the $\phi h^{4}$ contribution vanishes in the critical dimension $D=7$ relevant for the two-loop supergravity UV divergence, and the R-symmetry violation is circumvented.

\section{Conclusion and outlook}

In this paper the two-loop five-point amplitudes of both SYM and type II supergravity in ten dimensions were computed using the BRST cohomology method of [1-3]. The supersymmetric kinematic numerators are manifestly local, and their derivation follows an intuitive mapping between cubic graphs and superspace building blocks as guided by their BRST variation. Inspired by the BCJ-satisfying four-dimensional representation of [9], ten-dimensional numerators for all the planar and non-planar diagrams were written down in a form compatible with the color-kinematics duality.

The compatibility of the BRST principle (3.2) with the color-kinematics duality has already been encountered for tree-level $n$-point numerators [50] and one-loop five-point numerators [3]. Both of these cases emerge naturally from the field-theory limit of the corresponding superstring amplitudes, in the same way as the resulting BCJ subamplitude relations at tree-level [4] have an elegant derivation from string theory [51, 52]. This suggests that the superstring is a convenient starting point to understand the duality between color and kinematics in a broader context, see e.g. [53] for an example at reduced supersymmetry.

The string theory derivation of this work's results is an open problem since the genustwo five-point worldsheet correlator in [14] was determined only in the low-energy limit.

\footnotetext{
${ }^{9}$ The dimensional reduction of this component calculation is performed after expanding the contracted ten-dimensional Levi-Civita bilinears $\varepsilon^{m n_{1} n_{2} \ldots n_{9}} \varepsilon_{m p_{1} p_{2} \ldots p_{9}}=-9 ! \delta_{p_{1}}^{\left[n_{1}\right.} \delta_{p_{2}}^{n_{2}} \cdots \delta_{p_{9}}^{\left.n_{9}\right]}$ due to the contractions $\left\langle T_{\ldots}^{m}\right\rangle\left\langle\tilde{T}_{\ldots}^{m}\right\rangle$ between left- and right-moving superfields. If state 1 is chosen to be a $D$-dimensional dilaton, then the only dependence on $D$ stems from $e_{1} \cdot \tilde{e}_{1}=(D-2) \phi$.
} 
Still, the kinematic building blocks $T_{12,3 \mid 4,5}$ and $T_{1,2,3 \mid 4,5}^{m}$ have an alternative representation in [14] in variables of the non-minimal pure spinor formalism [28] which gives rise to the same component expansions when combined in a BRST-invariant manner. In particular, their appearance in the UV divergence (5.8) of the supergravity amplitude and the lowenergy limit of the closed superstring is identical, confirming the general expectation of [13]. Once the completion of the correlator in [14] beyond the low-energy limit is achieved, it would be desirable to reproduce the present field-theory amplitudes, using for example the techniques of [36]. Also, a derivation from the non-minimal pure spinor version of the ambitwistor string [54] would be desirable.

It would also be interesting to study the higher-point construction of the two-loop SYM amplitudes. In this case, a sequence of BRST-covariant tensorial building blocks is required to describe higher powers of loop momentum in $(n \geq 6)$-gon subdiagrams. At one loop, the analogous tensors have been found in [55] and used in the BRST cohomology derivation of the six-point one-loop SYM amplitude in [3]. Furthermore, the general form of the BRST principle (3.2) motivates to assemble higher-loop amplitudes in the same manner as described in this paper. The four-point BCJ representations at three and four loops in [5] and [56] are expected to provide valuable guidance. For the design of superspace numerators, the superfields of higher-mass dimensions constructed in [24] will play an essential role, and the low-energy limit of the three-loop superstring amplitude in [57] constrains the leading $\ell$-dependence in the numerators.

\section{Acknowledgments}

We thank Piotr Tourkine for valuable discussions and John-Joseph Carrasco as well as Henrik Johansson for helpful email correspondence. We are grateful to Piotr Tourkine for useful comments on the draft and to Humberto Gomez for collaboration on related topics. We acknowledge support by the European Research Council Advanced Grant No. 247252 of Michael Green. We would like to thank the organizers of the workshop "Superstring Perturbation Theory" held at Perimeter Institute in April 2015 for the opportunity to present this work. This research was supported in part by Perimeter Institute for Theoretical Physics. Research at Perimeter Institute is supported by the Government of Canada through Industry Canada and by the Province of Ontario through the ministry of Economic Development \& Innovation. O.S. is grateful to DAMTP for kind hospitality during initial steps of this work.

\section{A Diagrammatic bookkeeping of BRST variations}

In order to verify the BRST invariance of the integrands (4.7) and (4.15), it is convenient to devise a diagrammatic bookkeeping for the effect of the numerators' BRST variation on their associated loop integrals. This exploits the central requirement (3.2) that each summand in the BRST variation of a particular numerator must contain an inverse propagator of its associated integral. The need of redefining loop momenta to see the cancellation 
among different terms is bypassed once the remaining propagators are represented by a graph and manipulated through its automorphism symmetries.

To understand how this comes about, recall the BRST variation of the planar pentabox numerators $N_{1,2,3 \mid 4,5}^{(a)}(\ell)$ defined in (4.3),

$$
\begin{gathered}
Q N_{1,2,3 \mid 4,5}^{(a)}(\ell)=\frac{1}{2}\left(V_{1} T_{2,3 \mid 4,5}\left[\ell^{2}-\left(\ell-k_{1}\right)^{2}\right]+V_{2} T_{1,3 \mid 4,5}\left[\left(\ell-k_{1}\right)^{2}-\left(\ell-k_{12}\right)^{2}\right]\right. \\
\left.+V_{3} T_{1,2 \mid 4,5}\left[\left(\ell-k_{12}\right)^{2}-\left(\ell-k_{123}\right)^{2}\right]\right) .
\end{gathered}
$$

Therefore, the combined effect of the BRST variation on the product of $N^{(a)}$ and its associated penta-box propagators is given by

$$
\begin{aligned}
& \frac{2 Q N_{1,2,3 \mid 4,5}^{(a)}(\ell)}{\ell^{2} r^{2}(\ell+r)^{2}\left(\ell-k_{1}\right)^{2}\left(\ell-k_{12}\right)^{2}\left(\ell-k_{123}\right)^{2}\left(r-k_{5}\right)^{2}\left(r-k_{45}\right)^{2}} \\
& =\frac{1}{r^{2}(\ell+r)^{2}\left(r-k_{5}\right)^{2}\left(r-k_{45}\right)^{2}} \times\left\{\frac{V_{1} T_{2,3 \mid 4,5}}{\left(\ell-k_{1}\right)^{2}\left(\ell-k_{12}\right)^{2}\left(\ell-k_{123}\right)^{2}},\right. \\
& \left.\quad+\frac{-V_{1} T_{2,3 \mid 4,5}+V_{2} T_{1,3 \mid 4,5}}{\ell^{2}\left(\ell-k_{12}\right)^{2}\left(\ell-k_{123}\right)^{2}}+\frac{-V_{2} T_{1,3 \mid 4,5}+V_{3} T_{1,2 \mid 4,5}}{\ell^{2}\left(\ell-k_{1}\right)^{2}\left(\ell-k_{123}\right)^{2}}+\frac{-V_{3} T_{1,2 \mid 4,5}}{\ell^{2}\left(\ell-k_{1}\right)^{2}\left(\ell-k_{12}\right)^{2}}\right\},
\end{aligned}
$$

whose diagrammatic interpretation is given in the following figure:

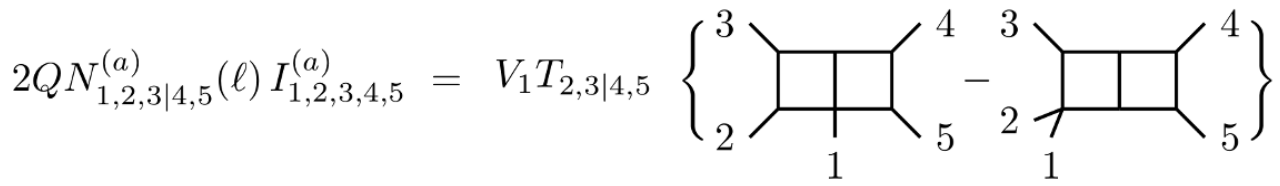

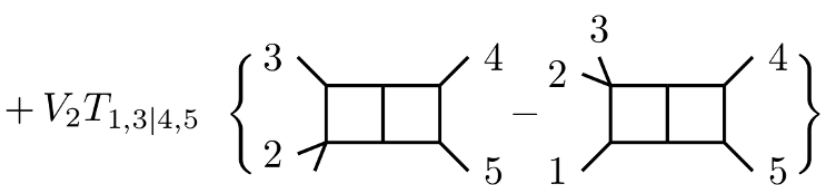

$$
\begin{aligned}
& 1
\end{aligned}
$$

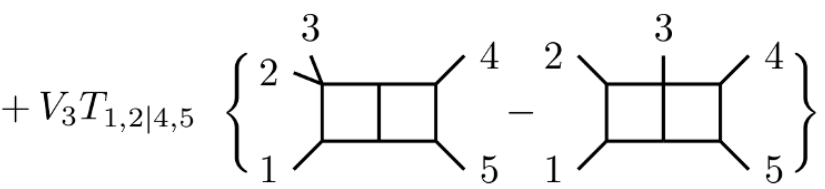

So it is clear that out of the six terms in (A.2) only two distinct integral topologies occur. Together with remaining graphs in figure 4, the BRST variation of the color-dressed fivepoint two-loop amplitude in (4.15) turns out to be captured by the five integral topologies $J^{\left(\alpha_{1}\right)}, \ldots, J^{\left(\alpha_{5}\right)}$ depicted in figure 8. Given the shorthands $I^{(a)}, \ldots, I^{(f)}$ in $(4.14)$, they 


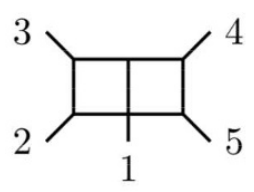

$J_{1,2,3,4,5}^{\left(\alpha_{1}\right)}$

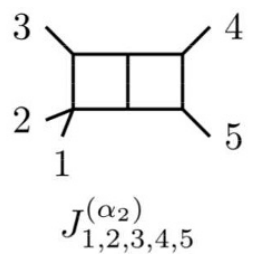

$J_{1,2,3,4,5}^{\left(\alpha_{2}\right)}$

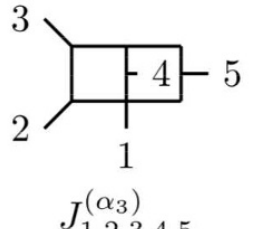

$J_{1,2,3,4,5}^{\left(\alpha_{3}\right)}$
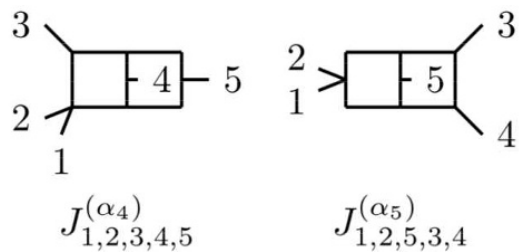

Figure 8. Five topologies of integrals occurring in the BRST variation of individual graphs

arise from the following BRST variations

$$
\begin{aligned}
2 Q N_{1,2,3 \mid 4,5}^{(a)}(\ell) I_{1,2,3,4,5}^{(a)}= & V_{1} T_{2,3 \mid 4,5}\left(J_{1,2,3,4,5}^{\left(\alpha_{1}\right)}-J_{1,2,3,4,5}^{\left(\alpha_{2}\right)}\right) \\
& +V_{2} T_{1,3 \mid 4,5}\left(J_{1,2,3,4,5}^{\left(\alpha_{2}\right)}-J_{2,3,1,5,4}^{\left(\alpha_{2}\right)}\right)+V_{3} T_{1,2 \mid 4,5}\left(J_{2,3,1,5,4}^{\left(\alpha_{2}\right)}-J_{3,4,5,1,2}^{\left(\alpha_{1}\right)}\right) \\
2 Q N_{1,2,3 \mid 4,5}^{(b)}(\ell) I_{1,2,3,4,5}^{(b)}= & V_{1} T_{2,3 \mid 4,5}\left(J_{1,2,3,4,5}^{\left(\alpha_{3}\right)}-J_{1,2,3,4,5}^{\left(\alpha_{4}\right)}\right) \\
& +V_{2} T_{1,3 \mid 4,5}\left(J_{1,2,3,4,5}^{\left(\alpha_{4}\right)}-J_{2,3,1,4,5}^{\left(\alpha_{4}\right)}\right)+V_{3} T_{1,2 \mid 4,5}\left(J_{2,3,1,4,5}^{\left(\alpha_{4}\right)}-J_{3,2,1,4,5}^{\left(\alpha_{3}\right)}\right) \\
2 Q N_{1,2|4,3| 5}^{(c)}(\ell, r) I_{1,2,3,4,5}^{(c)}= & V_{1} T_{2,5 \mid 3,4}\left(J_{1,3,4,5,2}^{\left(\alpha_{3}\right)}-J_{1,2,5,4,3}^{\left(\alpha_{5}\right)}\right) \\
& +V_{2} T_{1,5 \mid 3,4}\left(J_{1,2,5,4,3}^{\left(\alpha_{5}\right)}-J_{2,4,3,5,1}^{\left(\alpha_{3}\right)}\right)+V_{3} T_{4,5 \mid 1,2}\left(J_{3,1,2,5,4}^{\left(\alpha_{3}\right)}-J_{3,4,5,2,1}^{\left(\alpha_{5}\right)}\right) \\
& +V_{4} T_{3,5 \mid 1,2}\left(J_{3,4,5,2,1}^{\left(\alpha_{5}\right)}-J_{4,2,1,5,3}^{\left(\alpha_{3}\right)}\right)+V_{5} T_{1,2 \mid 3,4}\left(J_{5,4,3,1,2}^{\left(\alpha_{1}\right)}-J_{5,1,2,4,3}^{\left(\alpha_{1}\right)}\right) \\
2 Q N_{12,3 \mid 4,5}^{(d)} I_{1,2,3,4,5}^{(d)}= & \left(V_{1} T_{2,3 \mid 4,5}-V_{2} T_{1,3 \mid 4,5}\right) J_{1,2,3,4,5}^{\left(\alpha_{2}\right)} \\
2 Q N_{12,3 \mid 4,5}^{(e)} I_{1,2,3,4,5}^{(e)}= & \left(V_{1} T_{2,3 \mid 4,5}-V_{2} T_{1,3 \mid 4,5}\right) J_{1,2,3,4,5}^{\left(\alpha_{4}\right)} \\
2 Q N_{12,3 \mid 4,5}^{(f)} I_{1,2,3,4,5}^{(f)}= & \left(V_{1} T_{2,3 \mid 4,5}-V_{2} T_{1,3 \mid 4,5}\right) J_{1,2,3,4,5}^{\left(\alpha_{5}\right)},
\end{aligned}
$$

see figures 9 and 10 for a diagrammatic illustration of these identities. Using the BRST variations (A.3), the kinematic identities (3.9) and the automorphism symmetries

$$
\begin{array}{ll}
J_{1,2,3,4,5}^{\left(\alpha_{1}\right)}=J_{1,5,4,3,2}^{\left(\alpha_{1}\right)}, & J_{1,2,3,4,5}^{\left(\alpha_{2}\right)}=J_{2,1,3,4,5}^{\left(\alpha_{2}\right)}, \\
J_{1,2,3,4,5}^{\left(\alpha_{4}\right)}=J_{2,1,3,4,5}^{\left(\alpha_{4}\right)}=J_{1,2,3,5,4}^{\left(\alpha_{4}\right)}, & J_{1,2,3,4,5}^{\left(\alpha_{5}\right)}=J_{2,1,3,4,5}^{\left(\alpha_{3}\right)}=J_{1,2,3,4,4,4}^{\left(\alpha_{5}\right)}=J_{1,2,3,5,4}^{\left(\alpha_{3}\right)},
\end{array}
$$

one can show that (4.15) is BRST closed.

\section{B Comparison with the four-dimensional solution}

Just like the ten-dimensional numerators presented in the main text, the four-dimensional numerators written in table 1 of [9] are composed of scalar and vector building blocks. The numerators can be mapped into each other once the building blocks are replaced as ${ }^{10}$

$$
\begin{aligned}
& T_{12,3 \mid 4,5} \rightarrow \gamma_{12}\left(s_{45}-\frac{1}{2} s_{12}\right)+\frac{1}{4} s_{12}\left(\gamma_{23}-\gamma_{13}\right) \\
& T_{1,2,3 \mid 4,5}^{m} \rightarrow \gamma_{45}\left(k_{5}^{m}-k_{4}^{m}\right)+\frac{1}{2}\left[\gamma_{12}\left(k_{2}^{m}-k_{1}^{m}\right)+\gamma_{23}\left(k_{3}^{m}-k_{2}^{m}\right)+\gamma_{31}\left(k_{1}^{m}-k_{3}^{m}\right)\right],
\end{aligned}
$$

\footnotetext{
${ }^{10}$ The formal replacement rules in (B.1) do not imply that dimensional reduction of $\left\langle T_{12,3 \mid 4,5}\right\rangle$ and $\left\langle T_{1,2,3 \mid 4,5}^{m}\right\rangle$ yields the combinations of $\gamma_{i j}$ on the right hand sides.
} 


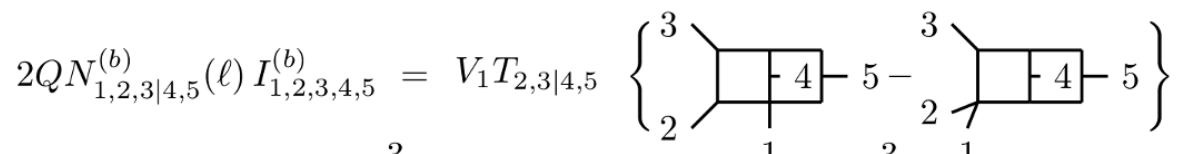

$$
\begin{aligned}
& +V_{2} T_{1,3 \mid 4,5}\left\{\begin{array}{l}
3 \\
2
\end{array}\right.
\end{aligned}
$$

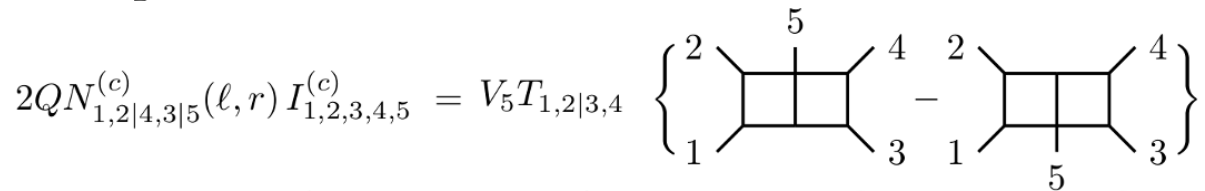

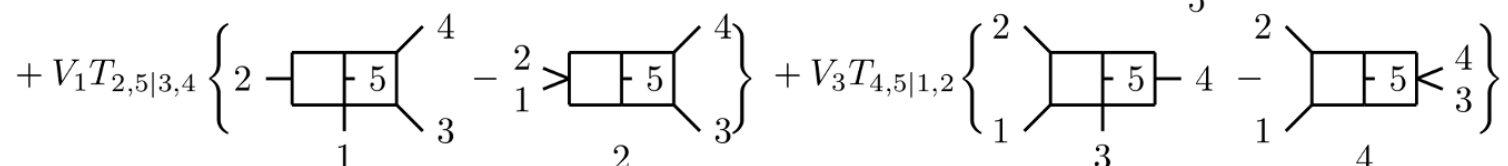

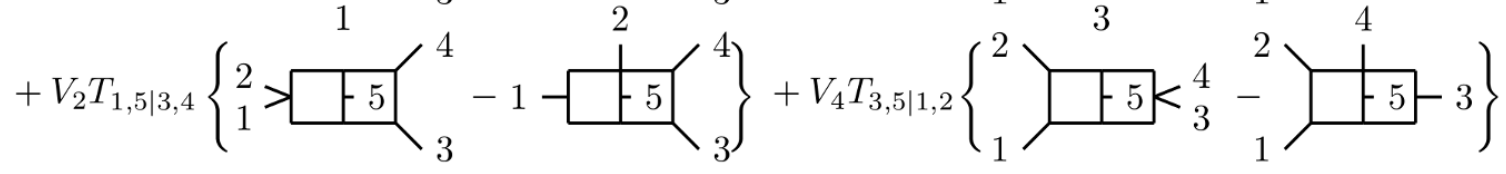

Figure 9. When multiplied by their corresponding integrands of (4.14), the BRST variations of the non-planar penta-box numerators $N_{1,2,3 \mid 4,5}^{(b)}$ and $N_{1,2,3 \mid 4,5}^{(c)}$ admit a diagrammatic interpretation.

$$
\begin{aligned}
& Q N_{12,3 \mid 4,5}^{(d)} I_{1,2,3,4,5}^{(d)}=\left(V_{1} T_{2,3 \mid 4,5}-V_{2} T_{1,3 \mid 4,5}\right) \\
& Q N_{12,3 \mid 4,5}^{(e)} I_{1,2,3,4,5}^{(e)}=\left(V_{1} T_{2,3 \mid 4,5}-V_{2} T_{1,3 \mid 4,5}\right) \\
& Q N_{12,3 \mid 4,5}^{(f)} I_{1,2,3,4,5}^{(f)}=\left(V_{1} T_{2,3 \mid 4,5}-V_{2} T_{1,3 \mid 4,5}\right)
\end{aligned}
$$

Figure 10. The diagrammatic interpretation for the BRST variation of the double-boxes after multiplication by their corresponding integrands given in (4.14).

where $\gamma_{i j}$ is built from spinor helicity expressions $[i j]$ and $\delta^{8}(\mathcal{Q})$ defined in [9],

$$
\gamma_{12} \equiv \delta^{8}(\mathcal{Q}) \frac{[12]^{2}[34][35][45]}{\varepsilon_{\mu \nu \lambda \rho} k_{1}^{\mu} k_{2}^{\nu} k_{3}^{\lambda} k_{4}^{\rho}} .
$$

The denominator is totally antisymmetric in $1,2, \ldots, 5$ and introduces a spurious singularity in the determinant $\operatorname{Det}\left(k_{i}^{\mu}\right)$ or the directed volume of the momenta $k_{1}^{\mu}, k_{2}^{\nu}, k_{3}^{\lambda}$ and $k_{4}^{\rho}$ with four-dimensional vector indices $\mu, \nu, \lambda, \rho$.

Given that this spinor helicity building block $\gamma_{i j}=-\gamma_{j i}$ satisfies

$$
\begin{aligned}
& 0=\gamma_{12}+\gamma_{13}+\gamma_{14}+\gamma_{15} \\
& 0=\left(\gamma_{12}+\gamma_{13}\right)\left(s_{23}-s_{45}\right)+\gamma_{23}\left(s_{12}-s_{13}\right)+\gamma_{45}\left(s_{14}-s_{15}\right)
\end{aligned}
$$


one can straightforwardly check that the superspace identities

$$
\begin{aligned}
& 0=\left\langle T_{12,3 \mid 4,5}+T_{12,4 \mid 5,3}+T_{12,5 \mid 3,4}\right\rangle \\
& 0=\left\langle T_{3,4,5 \mid 1,2}^{m}+T_{2,4,5 \mid 1,3}^{m}+T_{1,4,5 \mid 2,3}^{m}-T_{1,2,3 \mid 4,5}^{m}\right\rangle \\
& 0=\left\langle T_{15,2 \mid 3,4}+T_{25,1 \mid 3,4}+T_{35,4 \mid 1,2}+T_{45,3 \mid 1,2}-k_{m}^{5}\left(T_{3,4,5 \mid 1,2}^{m}+T_{1,2,5 \mid 3,4}^{m}\right)\right\rangle
\end{aligned}
$$

are all respected by the mapping in (B.1).

\section{One-loop UV divergences}

At one loop, the four-point UV divergences in SYM and supergravity are well-known from $[38,58]$. Using the pure spinor superspace representation of the five-point one-loop amplitudes in [3] and the identities in the appendix B of [23] we write the five-point UV divergence in terms of SYM tree amplitudes.

\section{C.1 One-loop UV divergences in SYM}

The counting of loop momenta is identical in SYM amplitudes at one- and two-loops, hence, the box diagrams dominate in the $(8-2 \epsilon)$ dimensional UV regime at one-loop via

$$
\int \frac{d^{8-2 \epsilon} \ell(2 \pi)^{2 \epsilon-8}}{\ell^{2}\left(\ell-k_{1}\right)^{2}\left(\ell-k_{12}\right)^{2}\left(\ell-k_{123}\right)^{2}}=\frac{i+\mathcal{O}(\epsilon)}{6(4 \pi)^{4} \epsilon} .
$$

The UV divergence in the critical dimension $D=8$ is characterized by a supersymmetrized $F^{4}$ counterterm [58]. From the pure spinor superspace representations of the four- and fivepoint one-loop amplitudes in SYM [3], one can extract the UV divergence,

$$
\begin{aligned}
\left.A^{1-\text { loop }}(1,2,3,4)\right|_{\mathrm{UV}} & =\frac{i}{6(4 \pi)^{4} \epsilon}\left\langle V_{1} T_{2,3,4}\right\rangle \\
\left.A^{1-\text { loop }}(1,2,3,4,5)\right|_{\mathrm{UV}} & =\frac{i}{6(4 \pi)^{4} \epsilon}\left\langle\frac{V_{12} T_{3,4,5}}{s_{12}}+\frac{V_{1} T_{23,4,5}}{s_{23}}+\frac{V_{1} T_{2,34,5}}{s_{34}}+\frac{V_{1} T_{2,3,45}}{s_{45}}+\frac{V_{51} T_{2,3,4}}{s_{51}}\right\rangle,
\end{aligned}
$$

see [3] for the scalar one-loop building blocks $T_{A, B, C}$. This reproduces the pure spinor analysis of $F^{4}$ amplitudes in [59], and the same matching can be found for the six-point one-loop amplitudes in [3]. An equivalent representation in terms of SYM tree amplitudes,

$$
\begin{aligned}
&\left.A^{1-\operatorname{loop}}(1,2,3,4)\right|_{\mathrm{UV}}=\frac{i}{6(4 \pi)^{4} \epsilon} s_{12} s_{23} A^{\text {tree }}(1,2,3,4) \\
&\left.\left(\begin{array}{l}
A^{1-\operatorname{loop}}(1,2,3,4,5) \\
A^{1-\operatorname{loop}}(1,3,2,4,5)
\end{array}\right)\right|_{\mathrm{UV}}=\frac{i}{6(4 \pi)^{4} \epsilon} P_{2} \cdot\left(\begin{array}{l}
A^{\text {tree }}(1,2,3,4,5) \\
A^{\text {tree }}(1,3,2,4,5)
\end{array}\right),
\end{aligned}
$$

reproduces the $\left(\alpha^{\prime}\right)^{2}$-correction to superstring tree amplitudes $[42,43]$ with $P_{2}$ defined by [41]

$$
P_{2} \equiv\left(\begin{array}{cc}
s_{12} s_{34}-s_{34} s_{45}-s_{51} s_{12} & s_{13} s_{24} \\
s_{12} s_{34} & s_{13} s_{24}-s_{24} s_{45}-s_{51} s_{13}
\end{array}\right)
$$


Together with the non-planar sector, the color-dressed one-loop amplitude with traceless gauge group generators $\operatorname{Tr}\left(t^{i}\right)=0$ gives rise to the UV divergence

$$
\begin{aligned}
\left.\mathcal{A}_{5}^{1-\text { loop }}\right|_{\mathrm{UV}}= & \frac{i N_{c}}{6(4 \pi)^{4} \epsilon}\left(\begin{array}{l}
\operatorname{Tr}\left(t^{1} t^{2} t^{3} t^{4} t^{5}\right) \\
\operatorname{Tr}\left(t^{1} t^{3} t^{2} t^{4} t^{5}\right)
\end{array}\right)^{T} \cdot P_{2} \cdot\left(\begin{array}{l}
A^{\text {tree }}(1,2,3,4,5) \\
A^{\text {tree }}(1,3,2,4,5)
\end{array}\right)+(2,3 \mid 2,3,4,5) \quad(\text { C. } .5) \\
& +\frac{i}{(4 \pi)^{4} \epsilon}\left\{\operatorname{Tr}\left(t^{1} t^{2} t^{3}\right) \operatorname{Tr}\left(t^{4} t^{5}\right) s_{45}\left[s_{24} A^{\text {tree }}(1,3,2,4,5)-s_{34} A^{\text {tree }}(1,2,3,4,5)\right]\right. \\
& +(4,5 \mid 1,2,3,4,5)\} .
\end{aligned}
$$

The kinematic factor along with the multitrace

$$
\left\langle\frac{V_{1} T_{23,4,5}}{s_{23}}+\frac{V_{12} T_{3,4,5}}{s_{12}}+\frac{V_{31} T_{2,4,5}}{s_{13}}\right\rangle=s_{45}\left[s_{24} A^{\text {tree }}(1,3,2,4,5)-s_{34} A^{\text {tree }}(1,2,3,4,5)\right]
$$

closely resembles the superfield structure of $(5.7)$ and was denoted by $C_{1 \mid 23,4,5}$ in $[23,59]$.

\section{C.2 One-loop UV divergences in supergravity}

The UV behavior of $n$-point supergravity amplitudes at one loop is affected by any $p$-gon diagram with $4 \leq p \leq n$. At four- and five-points, the leading UV divergence in dimensions $D=8-2 \epsilon$ can be assembled from scalar box integrals and tensor pentagon integrals. In the pure spinor representation of [3], this amounts to

$$
\begin{gathered}
\left.\mathcal{M}_{4}^{1-\text { loop }}\right|_{\mathrm{UV}}=\frac{i}{(4 \pi)^{4} \epsilon}\left|\left\langle V_{1} T_{2,3,4}\right\rangle\right|^{2}=\frac{i}{(4 \pi)^{4} \epsilon}\left|s_{12} s_{23} A^{\text {tree }}(1,2,3,4)\right|^{2} \\
\left.\mathcal{M}_{5}^{1-\text { loop }}\right|_{\mathrm{UV}}=\frac{i}{(4 \pi)^{4} \epsilon}\left\{\mid\left\langle\left. V_{1} T_{2,3,4,5}^{m}\right|^{2}+\left[\frac{\left|\left\langle V_{12} T_{3,4,5}\right\rangle\right|^{2}}{s_{12}}+(2 \leftrightarrow 3,4,5)\right]\right.\right. \\
\left.+\left[\frac{\left|\left\langle V_{1} T_{23,4,5}\right\rangle\right|^{2}}{s_{23}}+(2,3 \mid 2,3,4,5)\right]\right\} .
\end{gathered}
$$

This is the low-energy limit of closed-string one-loop amplitudes, see $[14,18]$ for the discussion of the five-point kinematic factor as well as (5.12) and (5.5) for $S_{0}$ and $M_{3}$, respectively. As discussed in section 5.3, the components with a $D$-dimensional dilaton and a graviton depend explicitly on the dimension $D$ via (5.13), and one gets

$$
\begin{array}{r}
\left|\left\langle V_{1} T_{2,3,4,5}^{m}\right\rangle\right|^{2}+\left[\frac{\left|\left\langle V_{12} T_{3,4,5}\right\rangle\right|^{2}}{s_{12}}+(2 \leftrightarrow 3,4,5)\right]+\left.\left[\frac{\left|\left\langle V_{1} T_{23,4,5}\right\rangle\right|^{2}}{s_{23}}+(2,3 \mid 2,3,4,5)\right]\right|_{\text {IIB }} ^{D} \\
=\left(\begin{array}{c}
\tilde{A}^{\text {tree }}(1,2,3,5,4) \\
\tilde{A}^{\text {tree }}(1,3,2,5,4)
\end{array}\right)^{T} \cdot S_{0} \cdot M_{3} \cdot\left(\begin{array}{c}
A^{\text {tree }}(1,2,3,4,5) \\
A^{\text {tree }}(1,3,2,4,5)
\end{array}\right) \times\left\{\begin{array}{c}
1 \\
: h^{5} \\
\frac{(8-D)}{6}: \phi h^{4}
\end{array}\right.
\end{array}
$$

The factor $(8-D) / 6$ in the $\phi h^{4}$ contribution implies that its five-point one-loop UV divergence in (C.7) vanishes in the critical dimension and does not violate the R-symmetry of type IIB supergravity, see the corresponding discussion along with the two-loop UV divergence in section 5.3. 
Open Access. This article is distributed under the terms of the Creative Commons Attribution License (CC-BY 4.0), which permits any use, distribution and reproduction in any medium, provided the original author(s) and source are credited.

\section{References}

[1] C.R. Mafra, Towards Field Theory Amplitudes From the Cohomology of Pure Spinor Superspace, JHEP 11 (2010) 096 [arXiv: 1007.3639] [INSPIRE].

[2] C.R. Mafra, O. Schlotterer, S. Stieberger and D. Tsimpis, A recursive method for SYM n-point tree amplitudes, Phys. Rev. D 83 (2011) 126012 [arXiv:1012.3981] [INSPIRE].

[3] C.R. Mafra and O. Schlotterer, Towards one-loop SYM amplitudes from the pure spinor BRST cohomology, Fortsch. Phys. 63 (2015) 105 [arXiv: 1410.0668] [INSPIRE].

[4] Z. Bern, J.J.M. Carrasco and H. Johansson, New Relations for Gauge-Theory Amplitudes, Phys. Rev. D 78 (2008) 085011 [arXiv:0805.3993] [InSPIRE].

[5] Z. Bern, J.J.M. Carrasco and H. Johansson, Perturbative Quantum Gravity as a Double Copy of Gauge Theory, Phys. Rev. Lett. 105 (2010) 061602 [arXiv: 1004.0476] [INSPIRE].

[6] N. Berkovits, Super Poincaré covariant quantization of the superstring, JHEP 04 (2000) 018 [hep-th/0001035] [INSPIRE].

[7] P.S. Howe, Pure spinors lines in superspace and ten-dimensional supersymmetric theories, Phys. Lett. B 258 (1991) 141 [Addendum ibid. B 259 (1991) 511] [InSPIRE].

[8] P.S. Howe, Pure spinors, function superspaces and supergravity theories in ten-dimensions and eleven-dimensions, Phys. Lett. B 273 (1991) 90 [INSPIRE].

[9] J.J. Carrasco and H. Johansson, Five-Point Amplitudes in $N=4$ super-Yang-Mills Theory and $N=8$ Supergravity, Phys. Rev. D 85 (2012) 025006 [arXiv:1106.4711] [InSPIRE].

[10] Z. Bern, T. Dennen, Y.-t. Huang and M. Kiermaier, Gravity as the Square of Gauge Theory, Phys. Rev. D 82 (2010) 065003 [arXiv: 1004.0693] [inSPIRE].

[11] C.R. Mafra and O. Schlotterer, PSS: From pure spinor superspace to components, http://www.damtp.cam.ac.uk/user/crm66/SYM/pss.html

[12] L. Brink, J.H. Schwarz and J. Scherk, Supersymmetric Yang-Mills Theories, Nucl. Phys. B 121 (1977) 77 [inSPIRE].

[13] M.B. Green, J.G. Russo and P. Vanhove, Ultraviolet properties of maximal supergravity, Phys. Rev. Lett. 98 (2007) 131602 [hep-th/0611273] [INSPIRE].

[14] H. Gomez, C.R. Mafra and O. Schlotterer, The two-loop superstring five-point amplitude and S-duality, arXiv:1504.02759 [INSPIRE].

[15] C.M. Hull and P.K. Townsend, Unity of superstring dualities, Nucl. Phys. B 438 (1995) 109 [hep-th/9410167] [INSPIRE].

[16] M.B. Green, M. Gutperle and H.-h. Kwon, Sixteen fermion and related terms in M-theory on $T^{2}$, Phys. Lett. B 421 (1998) 149 [hep-th/9710151] [INSPIRE].

[17] M.B. Green, Interconnections between type-II superstrings, $M$-theory and $N=4$ supersymmetric Yang-Mills, hep-th/9903124 [INSPIRE].

[18] M.B. Green, C.R. Mafra and O. Schlotterer, Multiparticle one-loop amplitudes and S-duality in closed superstring theory, JHEP 10 (2013) 188 [arXiv:1307.3534] [INSPIRE]. 
[19] E. Witten, Twistor-Like Transform in Ten Dimensions, Nucl. Phys. B 266 (1986) 245 [INSPIRE].

[20] J.P. Harnad and S. Shnider, Constraints and Field Equations for Ten-Dimensional Super Yang-Mills Theory, Commun. Math. Phys. 106 (1986) 183 [InSPIRE].

[21] P.A. Grassi and L. Tamassia, Vertex operators for closed superstrings, JHEP 07 (2004) 071 [hep-th/0405072] [INSPIRE].

[22] G. Policastro and D. Tsimpis, $R^{4}$, purified, Class. Quant. Grav. 23 (2006) 4753 [hep-th/0603165] [INSPIRE].

[23] C.R. Mafra and O. Schlotterer, Multiparticle SYM equations of motion and pure spinor BRST blocks, JHEP 07 (2014) 153 [arXiv: 1404.4986] [INSPIRE].

[24] C.R. Mafra and O. Schlotterer, Solution to the nonlinear field equations of ten dimensional supersymmetric Yang-Mills theory, Phys. Rev. D 92 (2015) 066001 [arXiv:1501.05562] [INSPIRE].

[25] C.R. Mafra, PSS: A FORM Program to Evaluate Pure Spinor Superspace Expressions, arXiv: 1007.4999 [INSPIRE].

[26] J.A.M. Vermaseren, New features of FORM, math-ph/0010025 [INSPIRE].

[27] N. Berkovits, Multiloop amplitudes and vanishing theorems using the pure spinor formalism for the superstring, JHEP 09 (2004) 047 [hep-th/0406055] [INSPIRE].

[28] N. Berkovits, Pure spinor formalism as an $N=2$ topological string, JHEP 10 (2005) 089 [hep-th/0509120] [INSPIRE].

[29] Z. Bern, L.J. Dixon, D.C. Dunbar and D.A. Kosower, One loop $n$ point gauge theory amplitudes, unitarity and collinear limits, Nucl. Phys. B 425 (1994) 217 [hep-ph/9403226] [INSPIRE].

[30] L.J. Dixon, Calculating scattering amplitudes efficiently, in proceedings of $Q C D$ and beyond, Boulder, Colorado, 4-30 June 1995, pp. 539-582 [hep-ph/9601359] [INSPIRE].

[31] N. Berkovits, Super-Poincaré covariant two-loop superstring amplitudes, JHEP 01 (2006) 005 [hep-th/0503197] [INSPIRE].

[32] N. Berkovits and C.R. Mafra, Equivalence of two-loop superstring amplitudes in the pure spinor and RNS formalisms, Phys. Rev. Lett. 96 (2006) 011602 [hep-th/0509234] [INSPIRE].

[33] H. Gomez and C.R. Mafra, The Overall Coefficient of the Two-loop Superstring Amplitude Using Pure Spinors, JHEP 05 (2010) 017 [arXiv: 1003.0678] [INSPIRE].

[34] C.R. Mafra, Pure Spinor Superspace Identities for Massless Four-point Kinematic Factors, JHEP 04 (2008) 093 [arXiv:0801.0580] [INSPIRE].

[35] Z. Bern, L.J. Dixon, D.C. Dunbar, M. Perelstein and J.S. Rozowsky, On the relationship between Yang-Mills theory and gravity and its implication for ultraviolet divergences, Nucl. Phys. B 530 (1998) 401 [hep-th/9802162] [INSPIRE].

[36] P. Tourkine, Tropical Amplitudes, arXiv:1309.3551 [INSPIRE].

[37] N. Marcus and A. Sagnotti, The Ultraviolet Behavior of $N=4$ Yang-Mills and the Power Counting of Extended Superspace, Nucl. Phys. B 256 (1985) 77 [INSPIRE].

[38] Z. Bern, J.J.M. Carrasco, L.J. Dixon, H. Johansson and R. Roiban, The Complete Four-Loop Four-Point Amplitude in $N=4$ super-Yang-Mills Theory, Phys. Rev. D 82 (2010) 125040 [arXiv: 1008.3327] [INSPIRE]. 
[39] A. Bilal, Higher derivative corrections to the nonAbelian Born-Infeld action, Nucl. Phys. B 618 (2001) 21 [hep-th/0106062] [INSPIRE].

[40] R. Medina, F.T. Brandt and F.R. Machado, The Open superstring five point amplitude revisited, JHEP 07 (2002) 071 [hep-th/0208121] [INSPIRE].

[41] O. Schlotterer and S. Stieberger, Motivic Multiple Zeta Values and Superstring Amplitudes, J. Phys. A 46 (2013) 475401 [arXiv:1205.1516] [inSPIRE].

[42] C.R. Mafra, O. Schlotterer and S. Stieberger, Complete N-Point Superstring Disk Amplitude I. Pure Spinor Computation, Nucl. Phys. B 873 (2013) 419 [arXiv:1106.2645] [InSPIRE].

[43] C.R. Mafra, O. Schlotterer and S. Stieberger, Complete N-Point Superstring Disk Amplitude II. Amplitude and Hypergeometric Function Structure, Nucl. Phys. B 873 (2013) 461 [arXiv:1106.2646] [INSPIRE].

[44] L.A. Barreiro and R. Medina, 5-field terms in the open superstring effective action, JHEP 03 (2005) 055 [hep-th/0503182] [INSPIRE].

[45] S. Stieberger and T.R. Taylor, Multi-Gluon Scattering in Open Superstring Theory, Phys. Rev. D 74 (2006) 126007 [hep-th/0609175] [INSPIRE].

[46] J. Brödel, O. Schlotterer, S. Stieberger, $\alpha^{\prime}$-expansion of open superstring amplitudes, http://mzv.mpp.mpg.de.

[47] H. Kawai, D.C. Lewellen and S.H.H. Tye, A Relation Between Tree Amplitudes of Closed and Open Strings, Nucl. Phys. B 269 (1986) 1 [inSPIRE].

[48] D.J. Gross and J.H. Sloan, The Quartic Effective Action for the Heterotic String, Nucl. Phys. B 291 (1987) 41 [InSPIRE].

[49] J.J.M. Carrasco, R. Kallosh, R. Roiban and A.A. Tseytlin, On the U(1) duality anomaly and the S-matrix of $N=4$ supergravity, JHEP 07 (2013) 029 [arXiv:1303.6219] [INSPIRE].

[50] C.R. Mafra, O. Schlotterer and S. Stieberger, Explicit BCJ Numerators from Pure Spinors, JHEP 07 (2011) 092 [arXiv:1104.5224] [INSPIRE].

[51] N.E.J. Bjerrum-Bohr, P.H. Damgaard and P. Vanhove, Minimal Basis for Gauge Theory Amplitudes, Phys. Rev. Lett. 103 (2009) 161602 [arXiv:0907.1425] [INSPIRE].

[52] S. Stieberger, Open \& Closed vs. Pure Open String Disk Amplitudes, arXiv:0907.2211 [INSPIRE].

[53] A. Ochirov and P. Tourkine, BCJ duality and double copy in the closed string sector, JHEP 05 (2014) 136 [arXiv:1312.1326] [INSPIRE].

[54] T. Adamo and E. Casali, Scattering equations, supergravity integrands and pure spinors, JHEP 05 (2015) 120 [arXiv:1502.06826] [INSPIRE].

[55] C.R. Mafra and O. Schlotterer, Cohomology foundations of one-loop amplitudes in pure spinor superspace, arXiv:1408.3605 [INSPIRE].

[56] Z. Bern, J.J.M. Carrasco, L.J. Dixon, H. Johansson and R. Roiban, Simplifying Multiloop Integrands and Ultraviolet Divergences of Gauge Theory and Gravity Amplitudes, Phys. Rev. D 85 (2012) 105014 [arXiv:1201.5366] [INSPIRE].

[57] H. Gomez and C.R. Mafra, The closed-string 3-loop amplitude and S-duality, JHEP 10 (2013) 217 [arXiv:1308.6567] [INSPIRE]. 
[58] M.B. Green, J.H. Schwarz and L. Brink, $N=4$ Yang-Mills and $N=8$ Supergravity as Limits of String Theories, Nucl. Phys. B 198 (1982) 474 [InSPIRE].

[59] C.R. Mafra and O. Schlotterer, The Structure of n-Point One-Loop Open Superstring Amplitudes, JHEP 08 (2014) 099 [arXiv: 1203.6215] [INSPIRE]. 